\title{
Pilot Beam Pattern Design for Channel Estimation in Massive MIMO Systems
}

\author{
Song Noh, Student Member, IEEE, Michael D. Zoltowski, Youngchul Sung, Senior Member, IEEE, and \\ David J. Love, Senior Member, IEEE
}

\begin{abstract}
In this paper, the problem of pilot beam pattern design for channel estimation in massive multiple-input multiple-output systems with a large number of transmit antennas at the base station is considered, and a new algorithm for pilot beam pattern design for optimal channel estimation is proposed under the assumption that the channel is a stationary Gauss-Markov random process. The proposed algorithm designs the pilot beam pattern sequentially by exploiting the properties of Kalman filtering and the associated prediction error covariance matrices and also the channel statistics such as spatial and temporal channel correlation. The resulting design generates a sequentially-optimal sequence of pilot beam patterns with low complexity for a given set of system parameters. Numerical results show the effectiveness of the proposed algorithm.
\end{abstract}

Index Terms-Channel estimation, massive MIMO systems, spatio-temporal correlation, training signal design.

\section{INTRODUCTION}

$\mathbf{M}$ ULTIPLE-INPUT multiple-output (MIMO) systems with large-scale transmit antenna arrays, so called massive MIMO systems, is one of the key technologies for future wireless communications. The large size of the transmit antenna array relative to the number of receive terminals can average out thermal noise, fast channel fading, and some interference, based on the law of large numbers [2], [3]. Massive MIMO provides high data rates and energy efficiency with simple signal processing because the propagation channels to terminal stations served by a base station equipped with massive MIMO are asymptotically orthogonal due to the increased beam resolution [4]. However, in practice, such benefits may be limited by channel estimation accuracy [2], [3]. This is especially true

Manuscript received September 13, 2013; revised January 15, 2014; accepted May 12, 2014. Date of publication May 30, 2014; date of current version September 11, 2014. This work was supported by the Basic Science Research Program through the National Research Foundation of Korea (NRF) funded by the Ministry of Education (2013R1A1A2A10060852). A preliminary version of this work was presented in [1], in which only the MISO case is considered. In this paper, the sequential design proposed in [1] is extended to the MIMO case, power allocation, and the block-fading case. Extensive simulation results with some realistic channel models are provided. The guest editor coordinating the review of this manuscript and approving it for publication was Prof. David Gesbert.

Y. Sung is with the Department of Electrical Engineering, Korea Advanced Institute of Science and Technology (KAIST), Daejeon 305-701, Korea (e-mail: ysung@ee.kaist.ac.kr).

S. Noh, M. D. Zoltowski, and D. J. Love are with the School of Electrical and Computer Engineering, Purdue University, West Lafayette, IN 47907 USA (e-mail: ssongnoh@purdue.edu; mikedz@ecn.purdue.edu; djlove@ecn.purdue. edu).

Color versions of one or more of the figures in this paper are available online at http://ieeexplore.ieee.org.

Digital Object Identifier 10.1109/JSTSP.2014.2327572 when full frequency reuse across neighboring cells is adopted; in this case, pilot contamination [2] leads to imperfect channel estimation which, in turn, yields severely degraded system performance. Furthermore, in contrast to the conventional MIMO system employing a small number of antennas, the overhead required for channel estimation for massive MIMO can be overwhelming and thereby severely limit the above mentioned benefits of massive MIMO. Since the available training resources are limited by either the channel coherence interval or the amount of interference induced by neighboring cells, fast and reliable channel estimation with reduced training overhead is critical to massive MIMO systems.

To tackle the challenge of channel estimation, much of the prior work focused on time-division duplex (TDD) operation assumed channel reciprocity [2], [3], [5], and reciprocity calibration [4] under the assumption of time-invariant channels within the coherence time. However, in most wireless systems, frequency-division duplex (FDD) operation is employed, and in this case the problem of channel estimation becomes more challenging because MIMO channel sounding requires substantial overhead (such as feedback and/or dedicated times for channel sounding) that scales with the number of antennas. Such overhead can limit the performance improvement that is expected in massive MIMO systems. There has been some work on channel estimation and channel state information (CSI) feedback techniques for FDD massive MIMO systems, based on compressive sensing [6], limited feedback [7], [8], and projected channels [9].

In this paper, we consider the problem of pilot beam design for downlink channel estimation in FDD massive MIMO systems, for the case where the number of symbol times for channel sounding within a channel coherence time is typically much less than the number of antennas. To design efficient pilot beam patterns, we here exploit channel statistics for massive MIMO systems derived from dynamic channel modelling [10]-[12] and analytical channel spatial correlation models [13]-[16]. Since the gain of beamforming in practical wireless systems is obtained mainly in slowly fading channels, we focus on slowly fading and exploit the correlated time-variations in the channel by adopting the widely-used Gauss-Markov channel model [17]. Under this model, the channel estimation performance can be enhanced through the use of optimal Kalman filtering and prediction that exploits the current and all previously received pilot signals, thereby shortening the required time for accurate channel estimation. Our model also incorporates spatial channel correlation that depends on both the antenna geometry and the scattering environment; 
experimental investigations and analytical studies have confirmed that this information is typically available in (massive) MIMO systems [4], [13]-[16], [18] and is locally ${ }^{1}$ time-wise stationary. By exploiting both the channel dynamics and the spatial correlation, we develop a low-complexity pilot beam pattern design procedure that provides a sequence of optimal pilot beam patterns that sequentially minimize the channel estimation mean square error (MSE) at each training instant based on a greedy approach. (The definition of sequential optimality will be provided soon.) The key idea underlying the proposed method is the joint use of spatio-temporal channel correlation and signal-to-noise ratio (SNR) combined with the exploitation of the structure of the error covariance matrices generated with optimal Kalman filtering under the Gauss-Markov model, to derive a sequence of optimal pilot beam patterns for each training period.

This paper is organized as follows: The system model and background are described in Section II. Section III describes the proposed pilot beam pattern design method. Practical issues of implementing the proposed method are discussed in Section IV. Numerical results are provided in Section V, followed by conclusions in Section VI.

Notation Vectors and matrices are written in boldface with matrices in capitals. All vectors are column vectors. For a matrix $\mathbf{A}, \mathbf{A}^{T}, \mathbf{A}^{H}$, and $\mathbf{A}^{*}$ indicate the transpose, Hermitian transpose, and complex conjugate of $\mathbf{A}$, respectively. $\operatorname{tr}(\mathbf{A})$ and $\operatorname{var}(\mathbf{A})$ denote the trace of $\mathbf{A}$ and the variance operator, respectively. $\operatorname{vec}(\mathbf{A})$ denotes the column vector obtained by stacking the elements of $\mathbf{A}$ columnwise. $[\mathbf{A}]_{i, j}$ denotes the element of $\mathbf{A}$ at the $i$-th row, and $j$-th column. $\operatorname{diag}\left(a_{1}, \ldots, a_{n}\right)$ denotes a diagonal matrix with diagonal elements $a_{1}, \ldots, a_{n}$, whereas $\operatorname{diag}(\mathbf{A})$ is the column vector containing the diagonal elements of a matrix $\mathbf{A}$. For a vector a, we use $\|\mathbf{a}\|_{1}$ for 1-norm and $\|\mathbf{a}\|_{2}$ for 2-norm. For two matrices $\mathbf{A}$ and $\mathbf{B}, \mathbf{A} \otimes \mathbf{B}$ denotes the Kronecker product, and $\mathbf{A} \preceq \mathbf{B}$ means that $\mathbf{B}-\mathbf{A}$ is positive semi-definite. $E\{\mathbf{x}\}$ represents the expectation of $\mathbf{x} . \mathbf{I}_{n}$ stands for the identity matrix of size $n$, and 1 denotes a column vector with all one elements. $\mathbb{R}_{+}$denotes the set of non-negative real numbers. $\iota=\sqrt{-1}$ is used for the imaginary number.

\section{SYSTEM ModeL}

\section{A. System Setup}

We consider a massive MIMO system with $N_{t}$ transmit antennas and $N_{r}$ received antennas $\left(N_{t} \gg N_{r}\right)$, where the channel is given by an $N_{r} \times N_{t}$ MIMO system with flat Rayleigh fading under the narrowband assumption (which easily extends to the case of wideband frequency-selective channel when the system adopts OFDM transmission [19]). The received signal at symbol time $k$ is given by

$$
\mathbf{y}_{k}=\mathbf{H}_{k} \mathbf{s}_{k}^{*}+\mathbf{w}_{k}, k=1,2, \ldots
$$

where $\mathbf{s}_{k}$ is the $N_{t} \times 1$ transmitted symbol vector at time $k, \mathbf{H}_{k}$ is the $N_{r} \times N_{t}$ MIMO channel matrix at time $k$, and $\mathbf{w}_{k}$ is the zeromean independent and identically distributed (i.i.d.) complex Gaussian noise vector at time $k$ with covariance matrix $\sigma_{w}^{2} \mathbf{I}_{N_{r}}$,

\footnotetext{
${ }^{1}$ It means that for a short period of time, the correlation characteristics do not change much.
}

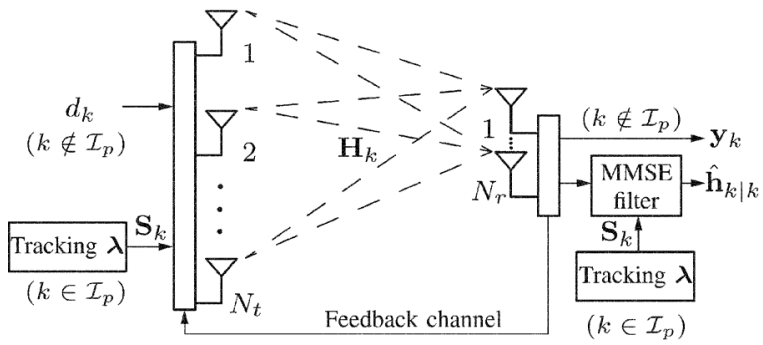

Fig. 1. Massive MIMO system model where $\boldsymbol{\lambda}$ is the eigenvalues of the prediction covariance matrix $\mathbf{P}_{k \mid k-1}$.

as shown in Fig. 1. (Here, we used the complex conjugate on $\mathbf{s}_{k}$ to keep the notation consistent with (7).)

1) MIMO Channel Correlation Model: For channel correlation, we consider the general Kronecker model that captures the transmit and receive antenna correlation [14], [15]. The transmit and receive channel covariance matrices reflect the geometry of the propagation paths and remain almost unchanged (locally time-wise) when compared to the rapidly-varying instant channel realization, since the array response to the scattering environments changes slowly compared to the user's location [20], [21]. Thus, the channel covariance matrices are assumed to be fixed over the considered time period for channel estimation, and the considered Kronecker channel model is given by

$$
\mathbf{H}_{k}=\mathbf{R}_{r}^{1 / 2} \tilde{\mathbf{H}}_{k}\left(\mathbf{R}_{t}^{1 / 2}\right)^{T},
$$

where $\left\{\tilde{\mathbf{H}}_{k} \in \mathbb{C}^{N_{r} \times N_{t}}, k=1,2, \ldots\right\}$ is an ergodic sequence of random matrices with independent zero-mean Gaussian elements with some variance, and $\mathbf{R}_{t} \in \mathbb{C}^{N_{t} \times N_{t}}$ and $\mathbf{R}_{r} \in \mathbb{C}^{N_{r} \times N_{r}}$ are deterministic transmit and receive correlation matrices, respectively, i.e., $\mathbf{R}_{t}=E\left\{\mathbf{H}_{k}^{H} \mathbf{H}_{k}\right\}$ and $\mathbf{R}_{r}=E\left\{\mathbf{H}_{k} \mathbf{H}_{k}^{H}\right\}$.

In the downlink training, the channel covariance matrices can be estimated by subspace estimation methods even without the knowledge of instantaneous channel state information [22]-[24], and there also exist methods that estimate the downlink channel covariance matrix using uplink training in FDD systems using techniques such as frequency calibration matrix [25], log-periodic array [26], or duplex array approach [27]. Furthermore, under some circumstances the channel covariance matrices $\mathbf{R}_{t}$ and $\mathbf{R}_{r}$ are approximately known a priori. For example, under the virtual channel condition [13], the use of uniform linear arrays (ULAs) at the transmitter and the receiver makes $\mathbf{R}_{t}$ and $\mathbf{R}_{r}$ approximately Toeplitz. By extending the one-ring model introduced by Jakes [19], the spatial correlation in the flat-fading case can be determined by the physical environment such as angle spread (AS), angle of arrival (AoA), and antenna geometry[14]. That is, in the case of a ULA with the AoA $\theta$ and the antenna spacing $\lambda D$, the channel covariance matrix is given by

$$
\left[\mathbf{R}_{t}\right]_{i, j}=\frac{\gamma}{2 \Delta} \int_{\theta-\Delta}^{\theta+\Delta} e^{-\iota 2 \pi D(i-j) \sin (\alpha)} d \alpha,
$$

where $\lambda$ is the wavelength, $\Delta$ is the AS, and $\gamma$ denotes propagation path loss defined in Section V. (This result can be extended to two-dimensional or planar arrays [16].) When the number of transmit antennas grows large, the eigenspace of $\mathbf{R}_{t}$ is closely 
approximated by a unitary Discrete Fourier Transform (DFT) matrix with the support of AoA distribution. Hereafter, we shall assume that the transmitter and the receiver have the knowledge of the channel covariance matrices. The assumption of known $\mathbf{R}_{t}$ will be revisited in Section IV.

2) Channel Variation in Time and Slotted Transmission Structure: For channel variation in time, we adopt a state-space model, i.e., the channel dynamic is given by the first-order stationary Gauss-Markov process [10]-[12], [28]

$$
\mathbf{h}_{k+1}=a \mathbf{h}_{k}+\sqrt{1-a^{2}} \mathbf{b}_{k+1}
$$

that satisfies the Lyapunov equation

$$
\mathbf{R}_{\mathbf{h}}=a^{2} \mathbf{R}_{\mathbf{h}}+\left(1-a^{2}\right) \mathbf{R}_{\mathbf{b}}
$$

and $\mathbf{R}_{\mathbf{h}}=E\left\{\mathbf{h}_{k} \mathbf{h}_{k}^{H}\right\}=\mathbf{R}_{\mathbf{b}}=E\left\{\mathbf{b}_{k} \mathbf{b}_{k}^{H}\right\}$ for all $k$ [20], where $\mathbf{h}_{k}:=\operatorname{vec}\left(\mathbf{H}_{k}\right), \mathbf{b}_{k}$ is a zero-mean and temporally independent plant Gaussian vector, and $a \in(0,1]$ is the temporal fading coefficient. ${ }^{2}$ (It is easy to verify that $\left\{\mathbf{h}_{k}, k=1,2, \ldots\right\}$ is a stationary process under this assumption.) The temporal fading correlation coefficient $a$ can be estimated [28]-[31], and we assume that $a$ is known. Then, under the Kronecker channel model (2) we have

$$
\mathbf{R}_{\mathbf{h}}=\mathbf{R}_{t} \otimes \mathbf{R}_{r}
$$

We assume slotted transmission with $M$ consecutive symbols as one slot which is comprised of a training period of $M_{p}$ symbols and a data transmission period of $M_{d}$ symbols so that $M=M_{p}+M_{d}$.

\section{B. Channel Estimation}

We consider the minimum mean square error (MMSE) approach for channel estimation [32] based on the current and all previous observations during training periods, i.e., $\hat{\mathbf{h}}_{k \mid k}:=$ $E\left\{\mathbf{h}_{k} \mid \mathbf{y}_{p}^{(k)}\right\}$ where $\mathbf{y}_{p}^{(k)}$ denotes all received signals during the pilot transmission up to symbol time $k$, given by

$$
\mathbf{y}_{p}^{(k)}=\left\{\mathbf{y}_{k^{\prime}} \mid k^{\prime} \leq k, k^{\prime} \in \mathcal{I}_{p}\right\}
$$

where $\mathcal{I}_{p}:=\left\{k=l M+m \mid l=0,1,2, \ldots, m=1, \ldots, M_{p}\right\}$. At each training symbol time, a pilot beam vector (or beam pattern) $\mathbf{s}_{k}$ of size $N_{t}, k \in \mathcal{I}_{p}$, is transmitted for channel estimation. During the data transmission period, on the other hand, the base station sends unknown data with transmit beamforming based on the estimated channel. ${ }^{3}$

Note that the received signal model (1) can be rewritten as

$$
\mathbf{y}_{k}=\mathbf{S}_{k}^{H} \mathbf{h}_{k}+\mathbf{w}_{k},
$$

${ }^{2}$ For Jakes' model, $a=J_{0}\left(2 \pi f_{D} T_{s}\right)$ [19], where $J_{0}(\cdot)$ is the zeroth-order Bessel function, $T_{s}$ is the transmit symbol interval, and $f_{D}$ is the maximum Doppler frequency shift.

${ }^{3}$ Transmit beamforming in FDD requires feedback information for channel state information (CSI) from the receiver. Thus, the quantized version of the downlink channel or the index of the quantized version of the channel chosen from a receiver can be fed back to the base station[33]. In addition, a quantized (or analog) version of the received training signal $\mathbf{y}_{k} \in \mathbb{C}^{N_{r}}$ can be fed back to enable channel estimation at the base station with a reduced amount of feedback [1]. The focus of the paper is not feedback quantization but optimal design of the pilot beam pattern for channel estimation. where $\mathbf{S}_{k}:=\mathbf{s}_{k} \otimes \mathbf{I}_{N_{r}}$ is an $N_{t} N_{r} \times N_{r}$ matrix. Then, we have a state-space model obtained from (4) and (7) and the optimal channel estimation is given by the Kalman filter for this statespace model [34]. During the training period, the Kalman filter performs a measurement update step for channel estimation at each symbol time, where the Kalman channel estimate and the related error covariance matrices are given by [34]

$$
\begin{aligned}
\hat{\mathbf{h}}_{k \mid k} & =\hat{\mathbf{h}}_{k k-1}+\mathbf{K}_{k}\left(\mathbf{y}_{k}-\mathbf{S}_{k}^{H} \hat{\mathbf{h}}_{k \mid k-1}\right) \\
\mathbf{P}_{k \mid k-1} & =a^{2} \mathbf{P}_{k-1 \mid k-1}+\left(1-a^{2}\right) \mathbf{R}_{\mathbf{h}}, \\
\mathbf{P}_{k \mid k} & =\mathbf{P}_{k \mid k-1}-\mathbf{K}_{k} \mathbf{S}_{k}^{H} \mathbf{P}_{k \mid k-1},
\end{aligned}
$$

where $\mathbf{K}_{k}=\mathbf{P}_{k \mid k-1} \mathbf{S}_{k}\left(\mathbf{S}_{k}^{H} \mathbf{P}_{k \mid k-1} \mathbf{S}_{k}+\sigma_{w}^{2} \mathbf{I}_{N_{r}}\right)^{-1}, \hat{\mathbf{h}}_{1 \mid 0}=$ $\mathbf{0}$, and $\mathbf{P}_{1 \mid 0}=\mathbf{R}_{\mathbf{h}}$. Here, $\mathbf{P}_{k \mid k}$ and $\mathbf{P}_{k \mid k-1}$ are the estimation and prediction error covariance matrices, respectively, defined as $\mathbf{P}_{k \mid k^{\prime}}=E\left\{\left(\mathbf{h}_{k}-\hat{\mathbf{h}}_{k \mid k^{\prime}}\right)\left(\mathbf{h}_{k}-\hat{\mathbf{h}}_{k \mid k^{\prime}}\right)^{H} \mid \mathbf{y}_{p}^{\left(k^{\prime}\right)}\right\}$, where $\hat{\mathbf{h}}_{k \mid k^{\prime}}:=E\left\{\mathbf{h}_{k} \mid \mathbf{y}_{p}^{\left(k^{\prime}\right)}\right\}$. During the data transmission period, the channel is predicted based on the last channel estimate of the previous training period as [34]

$$
\begin{aligned}
\hat{\mathbf{h}}_{l M+M_{p}+m \mid l M+M_{p}} & =a^{m} \hat{\mathbf{h}}_{l M+M_{p}+m \mid l M+M_{p}} \\
\mathbf{P}_{l M+M_{p}+m l M+M_{p}} & =a^{2 m} \mathbf{P}_{l M+M_{p} \mid l M+M_{p}}+\left(1-a^{2 m}\right) \mathbf{R}_{\mathbf{h}},
\end{aligned}
$$

where $m=1, \ldots, M_{d}$. During the data transmission period, the predicted channel can be used for transmit beamforming; for example, eigen-beamforming [2], [35] based on the predicted channel can be applied for maximum rate transmission.

In the simple case of multiple-input single-output (MISO) transmission, maximal ratio transmit beamforming based on the current channel estimate can be applied, and the transmit signal vector in this case is given by $\mathbf{s}_{k}=\hat{\mathbf{h}}_{k \mid l M+M_{p}} /\left\|\hat{\mathbf{h}}_{k \mid l M+M_{p}}\right\|_{2} d_{k}$, where $d_{k}$ is the data symbol at symbol time $k, k=l M+M_{p}+m$. From (7) and $\Delta \mathbf{h}_{k}:=\mathbf{h}_{k}-\hat{\mathbf{h}}_{k^{\prime} l M+M_{p}}$, the received signal model can be rewritten as

$$
y_{k}=\mathbf{s}_{k}^{H} \hat{\mathbf{h}}_{k \mid l M+M_{p}}+\mathbf{s}_{k}^{H} \Delta \mathbf{h}_{k}+w_{k} .
$$

The second term in (12) denotes the additional noise resulting from imperfect channel estimation. For simplicity, we adopt the deterministic ${ }^{4}$ approximation to $1 / N_{t}\left|\mathbf{s}_{k}^{H} \Delta \mathbf{h}_{k}\right|^{2}-1 / N_{t} \mathbf{s}_{k}^{H} \mathbf{P}_{k \mid l M+M_{p}} \mathbf{s}_{k} \underset{N_{t} \rightarrow \infty}{\stackrel{\text { a.s. }}{\longrightarrow}} 0[5]$. Then, the received SNR with the estimated channel is given by

$$
\text { Received SNR }=\frac{\left|\mathbf{s}_{k}^{H} \hat{\mathbf{h}}_{k \mid l M+M_{p}}\right|^{2}}{\mathbf{s}_{k}^{H} \mathbf{P}_{k^{\prime} l M+M_{p}} \mathbf{s}_{k}+\sigma_{w}^{2}} .
$$

\section{The Proposed Pilot Beam Pattern Design}

In this section, we present our proposed pilot beam pattern design methods that minimize the channel estimation MSE associated with optimal Kalman filtering explained in the previous section. The channel estimation MSE is directly related to the effective SNR [38] and thus such pilot beam pattern design can be leveraged to improve the training-based channel capacity.

\footnotetext{
${ }^{4} \mathrm{~A}$ rigorous $\mathrm{SNR}$ and achievable rate analysis for massive MIMO is available in [36], [37].
} 


\section{A. Greedy Sequential Design}

We notice from (11) that the channel estimation error during the data transmission period depends only on $a, \mathbf{R}_{\mathbf{h}}$ and the estimation error covariance matrix $\mathbf{P}_{l M+M_{p} \mid l M+M_{p}}$ at the last pilot symbol time. $a$ and $\mathbf{R}_{\mathbf{h}}$ are given, but the estimation MSE at the last pilot symbol time, $\operatorname{tr}\left(\mathbf{P}_{l M+M_{p} \mid l M+M_{p}}\right)$, can be minimized by properly designing the pilot beam pattern sequence $\left\{\mathbf{s}_{k} \mid k=l^{\prime} M+m, l^{\prime} \leq l, m=1, \ldots, M_{p}\right\}$. Here, since $\mathbf{P}_{l M+M_{p} l M+M_{p}}$ is a function of $\mathcal{S}:=\left\{\mathbf{s}_{j} \mid j=l^{\prime} M+m, m=\right.$ $\left.1, \ldots, M_{p}, j \leq l M+M_{p}\right\}, \mathcal{S}$ should be jointly optimized to minimize the MSE at time $k=l M+M_{p}$. However, this joint optimization is too complicated because the impact of $\mathcal{S}$ on $\mathbf{P}_{l M+M_{p} \mid l M+M_{p}}$ is intertwined over time. ${ }^{5}$ Furthermore, optimal channel estimation at $k=l M+M_{p}$ for some $l$ is not the only optimization goal since the MSE at $k=l^{\prime} M+M_{p}$ for each and every $l^{\prime}$ should be optimized for the $l^{\prime}$-th data transmission period. Therefore, we first adopt a greedy sequential optimization approach to design the pilot beam pattern sequence, which is formally stated as follows.

Problem 1: For each pilot symbol time $k$ starting from 1, given $\mathbf{s}_{j}$ for all pilot symbol time $j<k$, design $\mathbf{s}_{k}$ such that

$$
\begin{aligned}
& \min _{\mathbf{s}_{k}} \operatorname{tr}\left(\mathbf{P}_{k \mid k}\right) \\
& \text { s.t. }\left\|\mathbf{S}_{k}\right\|_{F}^{2}=N_{r}\left\|\mathbf{s}_{k}\right\|_{2}^{2}=N_{r} \rho_{p} .
\end{aligned}
$$

The solution to Problem 1 is given by the following proposition.

Proposition 1: Given all previous pilot signals $\mathbf{s}_{j}(j<k)$,

i) in the MISO case, the pilot beam pattern $\mathbf{s}_{k}$ at time $k$ minimizing $\operatorname{tr}\left(\mathbf{P}_{k \mid k}\right)$ is given by a scaled dominant eigenvector of the error covariance matrix $\mathbf{P}_{k \mid k^{\prime}}$ of the Kalman prediction for time $k$ [1], and

ii) in the MIMO case, if the Kalman prediction error covariance matrix $\mathbf{P}_{k^{\prime} k^{\prime}}$ for time $k$ is decomposed as

$$
\mathbf{P}_{k \mid k^{\prime}}=(\mathbf{U} \otimes \mathbf{V}) \operatorname{diag}\left(\boldsymbol{\Lambda}_{1}, \ldots, \boldsymbol{\Lambda}_{N_{t}}\right)(\mathbf{U} \otimes \mathbf{V})^{H}
$$

where $\mathbf{U} \in \mathbb{C}^{N_{t} \times N_{t}}$ and $\mathbf{V} \in \mathbb{C}^{N_{r} \times N_{r}}$ are unitary matrices, and $\Lambda_{i} \in \mathbb{R}_{+}^{N_{r} \times N_{r}}$ is a diagonal matrix with nonnegative real elements, ${ }^{6}$ then a locally optimal pilot beam pattern $\mathbf{s}_{k}$ at time $k$ for minimizing $\operatorname{tr}\left(\mathbf{P}_{k \mid k}\right)$ is given by a scaled version of a column vector of the unitary matrix $\mathbf{U}$ in (16).

Proof: See Appendix A.

Interestingly, it can be shown in the MISO case that the pilot beam pattern $\mathbf{s}_{k}$ obtained from (33) is equivalent to the first principal component direction of $\mathbf{P}_{k \mid k-1}$ given by

$$
\arg \max _{\left\|\mathbf{s}_{k}\right\|_{2}^{2}=\rho_{p}} \operatorname{var}\left(\mathbf{s}_{k}^{H}\left(\mathbf{h}_{k}-\hat{\mathbf{h}}_{k \mid k}\right)\right) .
$$

As seen in the proof, in the MIMO case, it is not easy to obtain a globally optimal solution, but the obtained locally optimal solution yields a nice property that can be exploited to derive an efficient pilot beam pattern design algorithm. Note that to

\footnotetext{
${ }^{5}$ The difficulty in applying standard dynamic programming (DP) [39] to the problem is that the contribution of $\mathbf{s}_{k}$ at time $k$ to the cost function is not localized at time $k$. It affects the so-called branch metric at time $k$ and all the following branch metrics.

${ }^{6}$ This assumption will be verified shortly in Proposition 2.
}

obtain the (sequentially) optimal $\mathbf{s}_{k}$, we need to perform the eigen-decomposition (ED) of $\mathbf{P}_{k \mid k^{\prime}}$ at each pilot symbol time $k$, and this can be computationally expensive since $N_{t}$ is large for massive MIMO systems. However, due to the following proposition regarding the eigen-space of the Kalman prediction error covariance matrix associated with Proposition 1, we can eliminate such heavy complexity burden when designing a sequentially optimal pilot beam pattern sequence.

Proposition 2: The Kalman filtering error covariance matrix $\mathbf{P}_{k \mid k}$ and the Kalman prediction error covariance matrix $\mathbf{P}_{k \mid k^{\prime}}$ generated by sequentially optimal $\mathbf{s}_{k}$ given by Proposition 1 are simultaneously diagonalizable with $\mathbf{R}_{\mathbf{h}}$ for any $k$ and $k^{\prime}(<k)$, under the assumption of $\mathbf{P}_{1 \mid 0}=\mathbf{R}_{\mathbf{h}}=\mathbf{R}_{t} \otimes \mathbf{R}_{r}{ }^{7}$

Proof: Proof is by induction. Let $\mathbf{R}_{t}=\mathbf{U} \mathbf{\Sigma} \mathbf{U}^{H}$ and $\mathbf{R}_{r}=$ $\mathbf{V} \Gamma \mathbf{V}^{H}$ be the ED of $\mathbf{R}_{t}$ and $\mathbf{R}_{r}$, respectively. Then, $\mathbf{P}_{1 \mid 0}=$ $(\mathbf{U} \otimes \mathbf{V}) \boldsymbol{\Lambda}^{(1)}(\mathbf{U} \otimes \mathbf{V})^{H}$, where $\boldsymbol{\Lambda}^{(1)}=\boldsymbol{\Sigma} \otimes \boldsymbol{\Gamma}$.

For any pilot symbol time $k=l M+m\left(m=1, \ldots, M_{p}\right)$, suppose that the Kalman prediction matrix for time $k$ is given by $\mathbf{P}_{k \mid k-1}=(\mathbf{U} \otimes \mathbf{V}) \boldsymbol{\Lambda}^{(k)}(\mathbf{U} \otimes \mathbf{V})^{H}$, where $\mathbf{U} \in \mathbb{C}^{N_{t} \times N_{t}}$ and $\mathbf{V} \in \mathbb{C}^{N_{r} \times N_{r}}$ are unitary matrices, and $\boldsymbol{\Lambda}^{(k)} \in \mathbb{R}^{N_{t} N_{r} \times N_{t} N_{r}}$ is a diagonal matrix given as

$$
\boldsymbol{\Lambda}^{(k)}=\operatorname{diag}\left(\boldsymbol{\Lambda}_{1}^{(k)}, \cdots, \Lambda_{N_{t}}^{(k)}\right) .
$$

By Proposition $1, \mathbf{s}_{k}$ is given by a scaled version of a column vector $\mathbf{u}_{i_{k}}$ of $\mathbf{U}$, i.e., $\mathbf{s}_{k}=\sqrt{\rho_{p}} \mathbf{u}_{i_{k}}$ with

$$
i_{k}:=\underset{i}{\arg \max } \operatorname{tr}\left\{\left(\rho_{p} \boldsymbol{\Lambda}_{i}^{(k)}+\sigma_{w}^{2} \mathbf{I}_{N_{r}}\right)^{-1} \rho_{p}\left(\boldsymbol{\Lambda}_{i}^{(k)}\right)^{2}\right\} .
$$

From the measurement update (10), $\mathbf{P}_{k \mid k}$ is given by

$$
\begin{aligned}
\mathbf{P}_{k \mid k}= & (\mathbf{U} \otimes \mathbf{V})\left\{\boldsymbol{\Lambda}^{(k)}-\left(\mathbf{e}_{i_{k}} \mathbf{e}_{i_{k}}^{T}\right) \otimes\right. \\
& {\left.\left[\rho_{p} \boldsymbol{\Lambda}_{i_{k}}^{(k)}\left(\rho_{p} \boldsymbol{\Lambda}_{i_{k}}^{(k)}+\sigma_{w}^{2} \mathbf{I}_{N_{r}}\right)^{-1} \boldsymbol{\Lambda}_{i_{k}}^{(k)}\right]\right\}(\mathbf{U} \otimes \mathbf{V})^{H} } \\
= & :(\mathbf{U} \otimes \mathbf{V}) \overline{\boldsymbol{\Lambda}}^{(k)}(\mathbf{U} \otimes \mathbf{V})^{H}
\end{aligned}
$$

where $\bar{\Lambda}^{(k)}$ is a diagonal matrix with nonnegative elements. (See Appendix B for details.) Thus, $\mathbf{P}_{k \mid k}$ and $\mathbf{P}_{k \mid k-1}$ are simultaneously diagonalizable. Since $\mathbf{R}_{\mathbf{h}}=\mathbf{R}_{t} \otimes \mathbf{R}_{r}=(\mathbf{U} \otimes$ $\mathbf{V}) \boldsymbol{\Lambda}^{(1)}(\mathbf{U} \otimes \mathbf{V})^{H}, \mathbf{P}_{k+1 \mid k}$ from the prediction step (9) is also simultaneously diagonalizable with $\mathbf{R}_{\mathbf{h}}$ since $\mathbf{P}_{k \mid k}$ is simultaneously diagonalizable with $\mathbf{R}_{\mathbf{h}}$.

Now consider a symbol time $k$ during the first data transmission period. In this case, the prediction error covariance matrix is given by

$$
\begin{aligned}
& \mathbf{P}_{M_{p}+m \mid M_{p}} \\
= & a^{2 m} \mathbf{P}_{M_{p} \mid M_{p}}+\left(1-a^{2 m}\right) \mathbf{R}_{\mathbf{h}} \\
= & (\mathbf{U} \otimes \mathbf{V})\left(\boldsymbol{\Lambda}^{(1)}-a^{2 m}\left(\boldsymbol{\Lambda}^{(1)}-\overline{\boldsymbol{\Lambda}}^{\left(M_{p}\right)}\right)\right)(\mathbf{U} \otimes \mathbf{V})^{H},
\end{aligned}
$$

where $m=1, \ldots, M_{d}$ and $\bar{\Lambda}^{\left(M_{p}\right)}$ is defined in (20). Thus, any prediction error covariance matrix during the first data period is simultaneously diagonalizable with $\mathbf{P}_{k \mid k}$ for $k \leq M_{p}$. Since this Kalman recursion repeats, we have the claim.

Note that the assumption (16) is valid under the Kronecker channel correlation model together with the pilot beam pattern selection proposed in Proposition 1. Proposition 2 states that all

\footnotetext{
${ }^{7}$ Such an initial parameter is a typical value for the Kalman filter, and there will be no loss [39].
} 
Kalman error covariance matrices under the sequentially optimal pilot beam pattern design have the same set of eigenvectors as $\mathbf{R}_{\mathbf{h}}$. This has an important practical implication: in each pilot transmission period, the base station transmits a pilot beam pattern at time $k$ chosen from a fixed set of orthogonal beam patterns, i.e., the transmit eigenvectors of $\mathbf{R}_{\mathbf{h}}$, according to some order depending on $\left\{\boldsymbol{\Lambda}_{i}^{(k)}, i=1, \cdots, N_{t}\right\}$ (defined in (18)). Note that (20) shows how a sequentially optimal pilot beam pattern at time $k$ reduces the channel estimation error by changing the eigenvalue distribution from $\boldsymbol{\Lambda}^{(k)}$ to $\overline{\boldsymbol{\Lambda}}^{(k)}$ with the measurement update step (only the $i_{k}$-th subblock is updated as $\left.\overline{\boldsymbol{\Lambda}}_{i_{k}}^{(k)}=\sigma_{w}^{2}\left(\rho_{p} \boldsymbol{\Lambda}_{i_{k}}^{(k)}+\sigma_{w}^{2} \mathbf{I}_{N_{r}}\right)^{-1} \boldsymbol{\Lambda}_{i_{k}}^{(k)}\right)$, and (21) shows how the eigenvalues of the channel prediction error covariance matrix change (from $\bar{\Lambda}^{(k)}$ to $\boldsymbol{\Lambda}^{(k+m)}$ ) during the pure prediction period. Exploiting these facts, we propose an efficient algorithm to obtain the sequence of sequentially optimal pilot beam patterns to minimize the channel estimation MSE at each symbol time. The algorithm is summarized in Algorithm 1.

\section{Algorithm 1 Sequentially Optimal Pilot Beam Pattern Design}

Require: Perform the ED of $\mathbf{R}_{t}=\mathbf{U} \boldsymbol{\Sigma} \mathbf{U}^{H}$ and $\mathbf{R}_{r}=\mathbf{V} \boldsymbol{\Gamma} \mathbf{V}^{H}$, and $\mathbf{R}_{\mathbf{h}}=\mathbf{R}_{t} \otimes \mathbf{R}_{r}$. Store $\boldsymbol{\lambda}^{(1)}=\operatorname{diag}(\boldsymbol{\Sigma} \otimes \boldsymbol{\Gamma})$, and $\mathbf{U}=\left[\mathbf{u}_{1}, \cdots, \mathbf{u}_{N_{t}}\right]$.

$$
\begin{aligned}
& \boldsymbol{\lambda}=\boldsymbol{\lambda}^{(1)} \text { and partition } \boldsymbol{\lambda}=\left[\boldsymbol{\lambda}_{1}^{T}, \cdots, \boldsymbol{\lambda}_{N_{t}}^{T}\right]^{T} \\
& \text { while } l=0,1, \cdots \text { do } \\
& \text { for } m=1 \text { to } M \text { do } \\
& k=l M+m \\
& \text { if } m \leq M_{p} \text { then } \\
& i_{k}=\operatorname{argmax}_{i} \sum_{j=1}^{N_{r}} \rho_{p} \lambda_{i j}^{2} / \rho_{p} \lambda_{i j}+\sigma_{w}^{2} \\
& \text { (See (19) and (39).) } \\
& \mathbf{s}_{k}=\sqrt{\rho_{p}} \mathbf{u}_{i_{k}} \\
& \boldsymbol{\lambda}_{i_{k}} \leftarrow \sigma_{w}^{2} \boldsymbol{\lambda}_{i_{k}} \cdot /\left(\rho_{p} \boldsymbol{\lambda}_{i_{k}}+\sigma_{w}^{2} \mathbf{1}\right)\left(\text { Step }^{*}\right) \\
& \text { end if } \\
& \boldsymbol{\lambda} \leftarrow a^{2} \boldsymbol{\lambda}+\left(1-a^{2}\right) \boldsymbol{\lambda}^{(1)}\left(\text { Step }^{* *}\right) \\
& \text { end for } \\
& \text { end while }
\end{aligned}
$$

(Here, ./ denotes the element-wise division and $\lambda_{i j}$ is the $j$-th element of $\boldsymbol{\lambda}_{i}$. Step ${ }^{*}$ incorporates the measurement update step (20) and Step ${ }^{* *}$ incorporates the prediction step (21).)
In Algorithm 1, the Kalman filtering error covariance matrix $\operatorname{tr}\left(\mathbf{P}_{k \mid k}\right)$ is minimized at each time $k$ with the hope that such a sequence minimizes the channel estimation MSE at the end of the pilot period of a slot. Since the important estimation measure is the estimation error at the end of the pilot period of each slot (which affects the channel estimation quality for the data transmission period under the time-varying channel assumption, as seen in (11)), we consider a modification to Algorithm 1 to design a pilot beam pattern sequence, targeting at the estimation error only at $l M+M_{p}$ for the $l$-th transmission block.

1) Problem 2: For each pilot symbol time $k=l M+m$ starting from 1, given $\mathbf{s}_{i}$ for all pilot symbol time $i<k$, design $\mathbf{s}_{k}$ such that

$$
\begin{aligned}
& \min _{\mathbf{s}_{k}} \operatorname{tr}\left(\mathbf{P}_{l M+M_{p} \mid k}\right) \\
& \text { s.t. }\left\|\mathbf{S}_{k}\right\|_{F}^{2}=N_{r}\left\|\mathbf{s}_{k}\right\|_{2}^{2}=N_{r} \rho_{p},
\end{aligned}
$$

where $l M+M_{p}$ is the end of the pilot period to which $k$ belongs.

Since we have

$$
\mathbf{P}_{l M+M_{p} \mid k}=a^{2\left(M_{p}-k\right)} \mathbf{P}_{k \mid k}+\left(1-a^{2\left(M_{p}-k\right)}\right) \mathbf{R}_{\mathbf{h}},
$$

the solution to Problem 2 is given by minimizing $\operatorname{tr}\left(\mathbf{P}_{k \mid k}\right)$ and Algorithm 1 can be used for this purpose too.

\section{B. Pilot Power Allocation}

In the pilot beam pattern design in Section III-A, we considered equal pilot power for each pilot symbol time. We relax the equal-power constraint here and consider the pilot beam pattern design problem again.

First, we will derive a necessary condition of an optimal pilot beam sequence that is useful for further pilot design. (This condition is given in Proposition 3.) To do so, let us first define some notations. For $1 \leq i \leq N_{t}$, let $\mathcal{K}_{i}=\left\{k \mid \mathbf{s}_{k}=\sqrt{\rho_{k}} \mathbf{u}_{i}\right\}=$ $\left\{k_{1}^{i}, k_{2}^{i}, \cdots, k_{\left|\mathcal{K}_{i}\right|}^{i}\right\} \subset\left\{l M+1, \ldots, l M+M_{p}\right\}$ be the time index set in the $l$-th slot for which the $i$-th transmit eigenvector $\mathbf{u}_{i}$ (obtained from $\mathbf{R}_{t}=\mathbf{U} \boldsymbol{\Sigma} \mathbf{U}^{H}$ and $\mathbf{U}=\left[\mathbf{u}_{1}, \cdots, \mathbf{u}_{N_{t}}\right]$ ) is used as the pilot beam pattern. Note that some eigenvectors may not be used as the pilot beam pattern depending on the channel statistics. Under the assumption that the transmitter has total power $M_{p} \rho_{p}$ for the pilot transmission period, we denote by $\rho_{k_{j}^{i}}$ the pilot signal power for the use of the $i$-th transmit eigenvector at time $k_{j}^{i} \in \mathcal{K}_{i}$ and define a pilot interval vector

$$
\begin{aligned}
\operatorname{tr}\left(\mathbf{P}_{l M+M_{p} \mid l M+M_{p}}\right)= & \sum_{i:\left|\mathcal{K}_{i}\right|=1} \operatorname{tr}\left(\overline{\boldsymbol{\Lambda}}_{i}^{\left(l M+M_{p}\right)}\right)+\sum_{i:\left|\mathcal{K}_{i}\right|=0} \operatorname{tr}\left(\overline{\boldsymbol{\Lambda}}_{i}^{\left(l M+M_{p}\right)}\right) \\
= & \sum_{i:\left|\mathcal{K}_{i}\right|=1} \operatorname{tr}\left(a^{2\left(l M+M_{p}-k^{i}\right)} \frac{\sigma_{w}^{2} \boldsymbol{\Lambda}_{i}^{\left(k^{i}\right)}}{\rho_{k^{i}} \boldsymbol{\Lambda}_{i}^{\left(k^{i}\right)}+\sigma_{w}^{2} \mathbf{I}_{N_{r}}}+\left(1-a^{2\left(l M+M_{p}-k^{i}\right)}\right) \boldsymbol{\Lambda}_{i}^{(1)}\right) \\
& +\sum_{i:\left|\mathcal{K}_{i}\right|=0} \operatorname{tr}\left(\overline{\boldsymbol{\Lambda}}_{i}^{\left(l M+M_{p}\right)}\right) \\
\propto & \sum_{i:\left|\mathcal{K}_{i}\right|=1} \operatorname{tr}\left(\frac{a^{2\left(l M+M_{p}-k^{i}\right)} \sigma_{w}^{2} \boldsymbol{\Lambda}_{i}^{\left(k^{i}\right)}}{\rho_{k^{i}} \boldsymbol{\Lambda}_{i}^{\left(k^{i}\right)}+\sigma_{w}^{2} \mathbf{I}_{N_{r}}}\right), \\
\text { where } \boldsymbol{\Lambda}_{i}^{\left(k^{i}\right)}= & a^{2\left(k^{i}-l M-1\right)} \boldsymbol{\Lambda}_{i}^{(l M+1)}+\left(1-a^{2\left(k^{i}-l M-1\right)}\right) \boldsymbol{\Lambda}_{i}^{(1)} .
\end{aligned}
$$




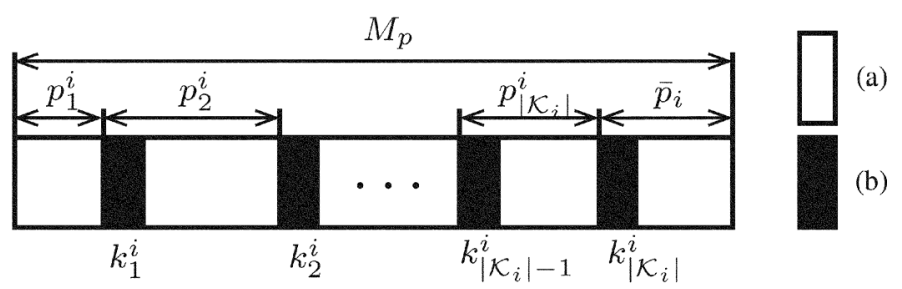

Fig. 2. The use of the $i$-th transmit eigenvector $\mathbf{u}_{i}$ as the pilot beam in a slot where $k_{j}^{i} \in \mathcal{K}_{i}$ and $\bar{p}_{i}=M_{p}-\left\|\mathbf{p}_{i}\right\|_{1}$ : (a) $\mathbf{u}_{i}$ is not used and (b) $\mathbf{u}_{i}$ is used.

$\mathbf{p}_{i}=\left[p_{1}^{i}, p_{2}^{i}, \cdots, p_{\left|\mathcal{K}_{i}\right|}^{i}\right]^{T}$ as shown in Fig. 2. The following proposition provides a property regarding optimal pilot power allocation.

Proposition 3: An optimal pilot beam pattern sequence minimizing $\operatorname{tr}\left(\mathbf{P}_{l M+M_{p} \mid l M+M_{p}}\right)$ in the $l$-th slot should satisfy the condition that all the pilot power for a transmit eigen-direction is allocated to the last use of the eigen-direction in the slot. That is, one transmit eigen-direction should not appear more than once in the pilot period of each slot.

Proof: See Appendix C.

Now consider the problem of joint design of beam pattern index selection and power allocation. As seen in Section III-A, the pilot beam pattern sequence design is a difficult problem even with fixed pilot power. In the case of pilot beam pattern sequence design with power control, we have a more complicated situation. Our approach to this complicated joint design problem is to separate the beam pattern index selection and the power allocation, although it is suboptimal. We again use the sequential beam pattern index selection based on (19) together with Proposition 3, but now we do not know the allocated pilot power beforehand. To circumvent this difficulty, we exploit the property of the argument in (19). Note that the argument in (19) is an increasing 8 function of $\boldsymbol{\Lambda}_{i}^{(k)}$ for any positive $\rho_{p}$. Hence, if we choose $\Lambda_{i^{\prime}}^{(k)}$ s.t. $\Lambda_{i^{\prime}}^{(k)} \succeq \Lambda_{i}^{(k)}$ for all $i \neq i^{\prime}$, this index $i^{\prime}$ is optimal. Note that for this selection method, we do not need the knowledge of the current pilot power $\rho_{k}$ at time $k$ ( $\rho_{p}$ in the case of (19)). However, there may not be such an index and hence, we replace this majorization criterion with a simple trace criterion since all the elements $\Lambda_{i}^{(k)}$ are non-negative. (Having the maximum trace is at least a necessary condition for being the majorizing index.) Based on this, we propose to choose the beam pattern index at time $k$ to minimize $\operatorname{tr}\left(\mathbf{P}_{k \mid k}\right)$ (or equivalently $\operatorname{tr}\left(\mathbf{P}_{l M+M_{p} \mid k}\right)$ as follows. First, consider time $k=l M+1$ under the assumption that the pilot sequence and power is already determined for the previous slots. We choose $i_{1}:=\arg \max _{i} \operatorname{tr}\left(\boldsymbol{\Lambda}_{i}^{(1)}\right)$. With the first index selected, consider $k=l M+2$. Now, applying the condition of Proposition 3 , we choose $i_{2}:=\arg \max _{i \notin\left\{i_{1}\right\}} \operatorname{tr}\left(\boldsymbol{\Lambda}_{i}^{(2)}\right)$. This is possible without knowing $\rho_{l M+1}$ since only $\boldsymbol{\Lambda}_{i_{1}}^{(2)}$ is affected by $\rho_{l M+1}$ and $i_{1}$ is not considered from $k \geq l M+2$. Then, we proceed to $k=l M+3$. In this way, we can choose $i_{1}, \cdots, i_{M_{p}}$ without knowing $\rho_{l M+1}, \cdots, \rho_{l M+M_{p}}$ based on the trace criterion and Proposition 3. For a selected index $i, \mathcal{K}_{i}=\left\{k_{1}^{i}\right\}$ and for an unselected index $i, \mathcal{K}_{i}=\emptyset$. Then, we have $\sum_{i=1}^{N_{t}}\left|\mathcal{K}_{i}\right| \leq M_{p}$.

\footnotetext{
${ }^{8} \mathrm{~A}$ real-valued function $\phi$ defined on some set $\mathcal{H}$ of $n \times n$ Hermitian matrices is increasing on $\mathcal{H}$ if $\mathbf{A} \preceq \mathbf{B} \Rightarrow \phi(\mathbf{A}) \leq \phi(\mathbf{B})$, whenever $\mathbf{A}, \mathbf{B} \in \mathcal{H}[40$ Ch. 16].
}

(Let us use $k^{i}$ for $k_{1}^{i}$.) Once $i_{1}, \cdots, i_{M_{p}}$ are determined, the optimization goal $\operatorname{tr}\left(\mathbf{P}_{l M+M_{p} \mid l M+M_{p}}\right)$ is given by (22).

Based on (22), the pilot power optimization problem is formulated as

$$
\begin{aligned}
& \min _{\boldsymbol{\rho}} \sum_{i:\left|\mathcal{K}_{i}\right|=1} \operatorname{tr}\left(\frac{a^{2\left(l M+M_{p}-k^{i}\right)} \sigma_{w}^{2} \boldsymbol{\Lambda}_{i}^{\left(k^{i}\right)}}{\rho_{k^{i}} \boldsymbol{\Lambda}_{i}^{\left(k^{i}\right)}+\sigma_{w}^{2} \mathbf{I}_{N_{r}}}\right) \\
& \text { s.t. }\|\boldsymbol{\rho}\|_{1}=M_{p} \rho_{p}, \rho_{k^{i}} \geq 0,
\end{aligned}
$$

where $\boldsymbol{\rho}=\left[\rho_{l M+1}, \ldots, \rho_{l M+M_{p}}\right]^{T}$. The problem (26) can be solved by water-filling power allocation[41] (see Appendix E for details), and the corresponding algorithm is summarized in Algorithm 2. In the MIMO case, $\boldsymbol{\rho}$ needs to be solved numerically from (60), whereas in the MISO case we have a closedform solution given by

$$
\rho_{k^{i}}=\left(a^{l M+M_{p}-k^{i}} \frac{\sigma_{w}}{\sqrt{\nu}}-\frac{\sigma_{w}^{2}}{\lambda_{i}^{\left(k^{i}\right)}}\right)^{+},
$$

where $(\cdot)^{+}=\max (\cdot, 0)$ and $\nu$ is evaluated from the power constraint (27).

$\overline{\text { Algorithm } 2 \text { Sequential Pilot Beam Pattern Design with Power }}$ Allocation

Require: Perform the ED of $\mathbf{R}_{t}=\mathbf{U} \boldsymbol{\Sigma} \mathbf{U}^{H}$ and $\mathbf{R}_{r}=\mathbf{V} \boldsymbol{\Gamma} \mathbf{V}^{H}$ where $\mathbf{R}_{\mathbf{h}}=\mathbf{R}_{t} \otimes \mathbf{R}_{r}$. Store $\boldsymbol{\lambda}^{(1)}=\operatorname{diag}(\boldsymbol{\Sigma} \otimes \boldsymbol{\Gamma})$, and $\mathbf{U}=\left[\mathbf{u}_{1}, \cdots, \mathbf{u}_{N_{t}}\right]$.

$$
\begin{aligned}
& \boldsymbol{\lambda}=\boldsymbol{\lambda}^{(1)} \text { and partition } \boldsymbol{\lambda}=\left[\boldsymbol{\lambda}_{1}^{T}, \cdots, \boldsymbol{\lambda}_{N_{t}}^{T}\right]^{T} \\
& \text { while } l=0,1, \cdots \text { do } \\
& \qquad \begin{aligned}
\mathcal{K}_{i}= & \emptyset \text { for } 1 \leq i \leq N_{t} \\
\text { for } & m=1 \text { to } M_{p} \text { do } \\
& k=l M+m \\
& i_{k}=\arg \max _{i \text { not used in this slot } \sum_{j} \lambda_{i j},} \\
& \text { where } \lambda_{i j} \\
& \text { is the } j \text {-th element of } \boldsymbol{\lambda}_{i}, \text { i.e., } \sum_{j} \lambda_{i j}=\operatorname{tr}\left(\boldsymbol{\Lambda}_{i}\right) . \\
& \text { Set } \mathcal{K}_{i k}=k \\
& \boldsymbol{\lambda} \leftarrow a^{2} \boldsymbol{\lambda}+\left(1-a^{2}\right) \boldsymbol{\lambda}^{(1)}
\end{aligned}
\end{aligned}
$$

end for

Obtain the power allocation $\boldsymbol{\rho}$ by solving (26).

$$
\begin{gathered}
\text { for } i=1 \text { to } N_{t} \text { do } \\
\text { if }\left|\mathcal{K}_{i}\right|=1 \text { then } \\
\mathbf{s}_{k^{i}}=\sqrt{\rho_{k^{i}}} \mathbf{u}_{i_{k}}
\end{gathered}
$$

end if

\section{end for}

for $m=1$ to $M$ do

Perform Kalman measurement update and prediction with the obtained $\left\{\mathbf{s}_{k}\right\}$ to track the correct error covariance matrix.

\section{end for}

\section{end while}

Note that in the first for-loop, the measurement update step is not implemented since we do not choose the used eigen-direction index again and thus we only need the prediction steps to select the eigen-direction indices. 
In high and low SNR regimes, the optimal power allocation can be approximated by simpler forms:

$$
\begin{aligned}
& \text { Case 1) High SNR: } \rho_{k^{i}} \lambda_{i j}^{\left(k^{i}\right)} \gg \sigma_{w}^{2} \\
& \qquad \rho_{k^{i}}=\frac{M_{p} \rho_{p}(1-a)}{1-a^{M_{p}}} a^{M_{p}-k^{i}},
\end{aligned}
$$

where $\lambda_{i j}^{\left(k^{i}\right)}$ is the $j$-th diagonal element of $\boldsymbol{\Lambda}_{i}^{\left(k^{i}\right)}$.

Case 2) Low SNR: $\rho_{k^{i}} \lambda_{i j}^{\left(k^{i}\right)} \ll \sigma_{w}^{2}$

$$
\begin{aligned}
\rho_{k^{i^{\prime}}} & =M_{p} \rho_{p} \\
i^{\prime} & =\arg \max _{i:\left|\mathcal{K}_{i}\right|=1} \operatorname{tr}\left(a^{2\left(l M+M_{p}-k^{i}\right)} \boldsymbol{\Lambda}_{i}^{\left(k^{i}\right)}\right) .
\end{aligned}
$$

In the special case of static channels, i.e., $a=1$, the proposed power allocation strategy covers the result of Kotecha and Sayeed [42], which considers the MMSE channel estimation with power control for quasi-static channels.

\section{Block-Fading Channel Model}

In this subsection, we consider a block Gauss-Markov fading channel model under which the channel is constant for each slot, i.e., $\mathbf{h}_{k}=\mathbf{h}_{l}$ for $k=l M+m(m=1,2, \cdots, M)$, but varies continuously across slots according to $\mathbf{h}_{l+1}=a \mathbf{h}_{l}+$ $\sqrt{1-a^{2}} \mathbf{b}_{l}$. We assume that the base station equipped with $N_{t}$ antennas serves a single-antenna terminal for simplicity [2]; each coherence time block of $M$ symbols is composed of a training period of $M_{p}$ symbols and a data transmission period of $M_{d}$ symbols; and $M_{p}<N_{t}$. By stacking $M_{p}$ symbols during the $l$-th training period, we have the received signal $\mathbf{y}_{l} \in \mathbb{C}^{M_{p}}$, given by

$$
\mathbf{y}_{l}=\mathbf{S}_{l}^{H} \mathbf{h}_{l}+\mathbf{w}_{l},
$$

where $\mathbf{y}_{l}=\left[y_{l M+1}, \ldots, y_{l M+M_{p}}\right]^{T}$ and $\mathbf{S}_{l}=$ $\left[\mathbf{s}_{l M+1} \cdots \mathbf{s}_{l M+M_{p}}\right]$. We further assume that $\mathbf{S}_{l}^{H} \mathbf{S}_{l}=\rho_{p} \mathbf{I}_{M_{p}}$ [38], [43]. The following proposition provides a property of optimal $\mathbf{S}_{l}$ under the block-fading channel model.

Proposition 4: Given all previous pilot signals $\mathbf{S}_{l^{\prime}}\left(l^{\prime}<l\right)$, the pilot beam signal $\mathbf{S}_{l}$ at the $l$-th training period minimizing $\operatorname{tr}\left(\mathbf{P}_{l \mid l}\right)$ is given by the scaled version of the $M_{p}$ dominant eigenvectors of the Kalman prediction error covariance matrix $\mathbf{P}_{l \mid l-1}$ for the $l$-th training period.

Proof: See Appendix D.

As in the symbolwise Gauss-Markov channel model, all Kalman prediction error covariance matrices that are used for the orthogonal pilot beam pattern design have the same set of eigenvectors of $\mathbf{R}_{\mathbf{h}}$, i.e., $\mathbf{R}_{\mathbf{h}}, \mathbf{P}_{l \mid l}$ and $\mathbf{P}_{l \mid l^{\prime}}$ are simultaneously diagonalizable. (Proof is omitted since it can be shown similarly as in Proposition 2.) Thus, the proposed algorithm in the previous section can easily be extended to the block-fading Gauss-Markov channel model. Previously, it was proposed by some other researchers that the $M_{p}$ dominant eigenvectors of $\mathbf{R}_{\mathbf{h}}$ are used for the $M_{p}$ pilot symbol times for every slot under the block i.i.d. fading model [42]. However, in our proposed method, we use for the $M_{p}$ pilot beam patterns in the $l$-th slot the $M_{p}$ dominant eigenvectors of $\mathbf{P}_{l \mid l^{\prime}}$ instead of $\mathbf{R}_{\mathbf{h}}$ to incorporate channel dynamics and to track the most efficient $M_{p}$ eigen-directions over time. Note that the full set of eigenvectors is the same for $\mathbf{R}_{\mathbf{h}}$ and $\mathbf{P}_{l \mid l^{\prime}}$ and that $\mathbf{R}_{\mathbf{h}}$ does not change over time under the considered stationary Gauss-Markov channel model. The tracking feature of the proposed method yields a significant gain over the previous method in time-varying channels when the channel dynamic is known, as seen in Section V. Some progresses on this approach are reported by relaxing the orthogonality condition [44] and designing structured training codebook [45].

\section{DisCuSSION: PRACTICAL IMPLEMENTATION AND MULTI-USER SCENARIO}

In this section, we make some comments relative to practical implementation of our proposed pilot design and channel estimation scheme in real-world massive MIMO systems.

First, consider the type and amount of feedback necessary for a massive MIMO system. One approach is to have the mobile station estimate the full channel state vector and feed that back to the base station. For a massive MIMO system, this approach requires a large amount of feedback and may be difficult to implement in practice. Alternatively, the mobile station may simply feed back the received signal $\mathbf{y}_{k} \in \mathbb{C}^{N_{r}}$ at each time instant, i.e., have the mobile station effectively transmit back the inner product between the current beamforming vector and the current channel state vector plus noise, and use that information to form an estimate of the channel at the base station [1]. The latter method is more effective in terms of the amount of feedback and does not require any modifications to the algorithm proposed in this paper.

Second, consider the estimation of the channel fading coefficient $a$ in the channel time-varying model (4). Since $a$ depends on the mobile speed of the receiver, it can be estimated by using the uplink received signal directly [28]-[31]. (A simple correction due to the uplink and downlink carrier frequency difference in FDD systems should be applied.) This problem falls into the general area of system identification of state-space models. Especially, blind techniques based on subspace approaches can be applied here [31].

Next, throughout the paper, we assume that the downlink channel covariance matrix $\mathbf{R}_{\mathbf{h}}$ is known to the system for a general channel model. If $\mathbf{R}_{\mathbf{h}}$ is estimated at the receiver (mobile station) and fed back to the base station through some control channel, the feedback overhead may be significant. Fortunately, there exist methods that can circumvent this difficulty. One way is to estimate the downlink channel covariance matrix $\mathbf{R}_{\mathbf{h}}$ from the uplink channel covariance matrix [25]-[27]. ${ }^{9}$ The downlink $\mathbf{R}_{\mathbf{h}}$ can be estimated from the uplink channel covariance matrix even though they are a bit separated in the frequency domain in the FDD case. Interested readers are referred to [25]-[27].

Furthermore, we here propose even a simpler method to obtain $\mathbf{R}_{\mathbf{h}}$ based on the one ring model and the Toeplitz distribution theorem for 1-dimensional or 2-dimenional large uniform arrays. Consider a 1-dimensional large uniform array with $N_{t}$ antenna elements for simplicity. Each element of the array performs spatial-sampling of the signal. Thus, if we view these spatial samples as discrete-time samples, the conventional (discrete-time) frequency domain corresponds to the virtual angle

\footnotetext{
${ }^{9}$ Note that in the MISO downlink case, the uplink is SIMO. In the time-domain duplex (TDD) case, the uplink and downlink channel covariance matrices are the same.
} 
domain. ${ }^{10}$ For the one-ring model with a uniform array under a far-field assumption, the channel covariance matrix $\mathbf{R}_{\mathbf{h}}$ is Toeplitz [16]. It is known that when the size of a Toeplitz covariance matrix is large, the Toeplitz matrix can be eigen-decomposed by a DFT matrix, which is known as the Toeplitz distribution theorem [16], [46], [47], i.e., $\mathbf{R}_{\mathbf{h}} \approx \mathbf{F} \mathbf{D F}{ }^{H}$ where $\mathbf{F}$ is a DFT matrix and $\mathbf{D}$ is a diagonal matrix that contains the virtual angular power spectral values. (This is why the eigen-decomposition of a Toeplitz covariance matrix is also called the spectral decomposition.) For a one-ring model with angle-of-arrival (AoA) and angle-dispersion $(\Delta)$, the elements of $\mathbf{D}$ are non-zero only for the angle spectrum $(\mathrm{AoA}-\Delta$, AoA $+\Delta)$. Thus, when AoA and $\Delta$ are given, $\mathbf{R}_{\mathbf{h}}$ can be constructed from the corresponding columns of $\mathbf{F}$ and the angular power spectral values. Note that the $k$-th column of $\mathbf{F}$ is given by

$$
\frac{1}{\sqrt{N_{t}}}\left[1, e^{\iota 1 \xi_{k} 2 \pi / N_{t}}, \cdots, e^{\iota\left(N_{t}-1\right) \xi_{k} 2 \pi / N_{t}}\right]^{H} .
$$

This is simply the steering vector for the physical angle $\theta_{k}=\sin ^{-1}\left(\xi_{k} \lambda / d\right)$. Under the model, the channel is given by a random linear combination of column vectors or steering vectors with the form (32) looking at the angle range $(\mathrm{AoA}-\Delta, \operatorname{AoA}+\Delta)$. (Channel estimation in the previous sections is nothing but estimation of the random linear combination coefficients.) The AoA can be estimated from the uplink signal model (there are numerous practical AoA or DoA estimation algorithms) and $\Delta$ can be pre-measured or predetermined for each carrier frequency by reflecting the typical scattering environment. The angular power spectrum can also be estimated based on one of typical spectral estimation methods [48]. Here, the angular power spectrum is estimated by using the uplink signal and a correction similar to those in [25]-[27] can be applied to obtain a downlink counterpart. Simulations will be presented towards the end of the next section in which the pilot beam patterns are approximated by DFT vectors without much loss in performance.

In summary, the proposed pilot design and channel estimation method can be run in the following practical way:

1) first estimate the AoA based on the uplink signal and selects the columns of $\mathbf{F}$ corresponding to $(\mathrm{AoA}-\Delta, \mathrm{AoA}+$ $\Delta)$

2) estimate the angular power profile for $(\mathrm{AoA}-\Delta$, AoA + $\Delta$ ) from the uplink channel response [48], and finally obtain a downlink power profile via correction [25]-[27]. This downlink angular power profile gives $\boldsymbol{\lambda}^{(1)}$ in Algorithm $1 ; 11$

3) estimate the mobile speed of the terminal (i.e., a) based on the uplink by using one of system identification algorithms [28]-[31]; and

\footnotetext{
${ }^{10}$ The virtual angle $\xi$ is related to the physical angle $\theta$ by $\xi=d / \lambda \sin (\theta)$, where $d$ is the antenna spacing and $\lambda$ is the carrier wavelength. When $d / \lambda=$ $1 / 2,-\pi / 2 \leq \theta \leq \pi / 2$ corresponds to $-1 / 2 \leq \xi \leq 1 / 2$.

${ }^{11}$ The simple assumption of wide-sense stationarity may not hold in real wireless channels. However, wireless channels are usually characterized by local quasi-stationarity [49], as per empirical measurements conducted under urban macrocell conditions [50] (i.e., within local quasi-stationary time periods, the proposed method can operate with a window-based average to update the channel statistics of low-mobility users).
}

4) finally run one of the algorithms in the previous sections. (By reciprocity, the AoA and the terminal velocity are the same for the up and down links.)

Finally, consider the multi-user case. Note that the system model (1) is for a single-user MIMO channel. However, many of current real-world wireless communication systems as those in 3GPP support user-dedicated pilot and control channels in addition to a common pilot and control channel for effective channel estimation for each user. Thus, the proposed method can be applied to these dedicated pilot channels. Furthermore, the proposed method can well be combined with the recently proposed joint spatial division and multiplexing (JSDM) framework for multiuser massive MIMO systems [16]. In the JSDM, the multiple users (MU) in a sector are partitioned into groups each of which has approximately the same channel covariance matrix. (Each set of the partition can be viewed as a virtual subsector.) Here, if the groups or subsectors are sufficiently well separated in the AoA domain, the dominant eigenvectors of the channel covariance matrices become linearly independent for different groups. To serve MU-MIMO in the same time-frequency slot, we can choose the users that have non-overlapping supports of their AoA distribution as in [16]. Then, the optimal pilot beam patterns become different and orthogonal among non-overlapping groups. In this case, the system model (1) can be regarded as the signal model for a scheduled user in one of the non-overlapping subsectors of the overall multi-user downlink.

\section{NuMERICAL RESUlTS}

In this section, we provide some numerical results to evaluate the performance of the proposed algorithms. We considered $N_{t} \in\{32,250\}$ transmit antennas and $N_{r} \in\{1,2\}$ receive antennas for our massive MIMO systems. We adopted $2.5 \mathrm{GHz}$ carrier frequency and $100 \mu \mathrm{s}$ symbol duration with a typical mobile speed range from $v=3 \mathrm{~km} / \mathrm{h}(a=0.9999)$ to $30 \mathrm{~km} / \mathrm{h}(a=0.9995)$. For all considered pilot design methods, we used Kalman filtering and prediction for the channel estimator. To evaluate the channel estimation performance, we computed the normalized mean square error (NMSE), given by $\operatorname{tr}\left(\mathbf{P}_{k \mid k}\right) / \operatorname{tr}\left(\mathbf{R}_{\mathbf{h}}\right)$. The pilot symbol SNR was defined as $\rho_{p} / \sigma_{w}^{2}$, the data symbol SNR was defined as $\rho_{d} / \sigma_{w}^{2}$, and the two SNR values were the same throughout the simulation. The noise variance $\sigma_{w}^{2}$ was determined according to the SNR value with $\rho_{p}=\rho_{d}=1$, and the received SNR is defined as (13), which incorporates the effect of beamforming gain and imperfect channel estimation. The channel estimation performance for each of the considered methods was averaged over 1,000 Monte Carlo runs.

First, we considered the exponential correlation model for channel spatial correlation, given by $\left[\mathbf{R}_{t}\right]_{i, j}=r_{t}^{2|i-j|}$ and $\left[\mathbf{R}_{r}\right]_{i, j}=r_{r}^{2|i-j|}$, where $r_{t}$ and $r_{r}$ are the transmit and receive correlation coefficients between two adjacent antenna elements, respectively $\left(r_{t}=r_{r}=\bar{r}\right.$ for simplicity). Since the phase of $\bar{r}$ is irrelevant to the eigenvalues of $\mathbf{R}_{\mathbf{h}}$, we assume without loss of generality that the phase of $\bar{r}$ is fixed to be zero (i.e., $\bar{r} \in \mathbb{R}$ ). Fig. 3 shows the channel estimation performance of several pilot pattern design methods [43] for the exponential channel correlation model with $\bar{r}=0.6, N_{t}=32$, and $N_{r}=2$ 

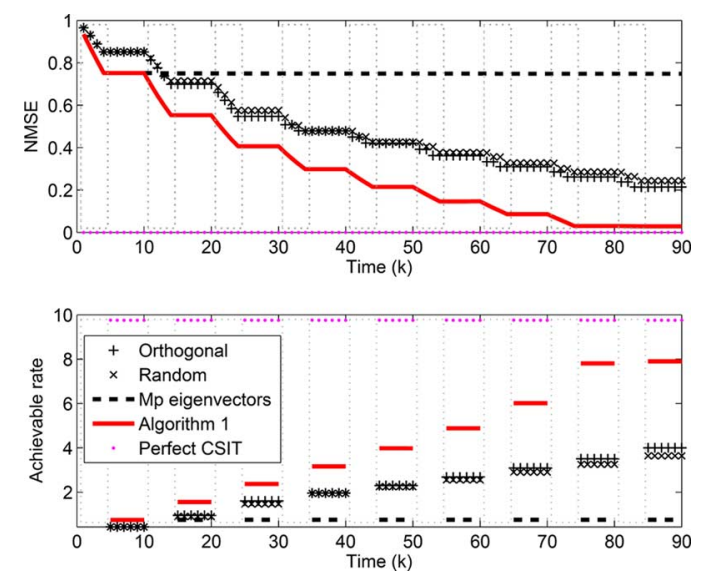

Fig. 3. NMSE and a lower bound on achievable rate $(\mathrm{bit} / \mathrm{s} / \mathrm{Hz})$ versus time index $k: M=10, M_{p}=4, \sigma_{w}^{2}=10^{-1.5}, N_{t}=32, N_{r}=2, \bar{r}=0.6$, and $v=3 \mathrm{~km} / \mathrm{h}$ (The dotted rectangles denote pilot transmission periods.).

(without path loss). The performance of the $M_{p}$ dominant eigenvectors of $\mathbf{R}_{\mathbf{h}}$ as the $M_{p}$ pilot beam patterns for every pilot period is also shown. It is seen that the proposed algorithm tracks the channel state fast due to the ability of the proposed method's tracking the spectral distribution of the channel MSE. Thus, the proposed method converges more quickly. The use of orthogonal or random beam patterns (which span the overall space) yields reasonable performance with slightly increased convergence time compared to the proposed method. In the case of the fixed $M_{p}$ dominant eigenvectors of $\mathbf{R}_{\mathbf{h}}$ for the pilot beam pattern in every pilot period, one can only minimize the channel MSE along the fixed $M_{p}$ eigen-directions, and the coverage of only $M_{p}$ fixed eigen-directions in the space is not enough for very large $N_{t}$ when $M_{p}$ is small. Hence, the channel estimation MSE performance of the fixed pilot beam pattern method is saturated quickly. By replacing the channel estimation error plus noise with independent additive Gaussian noise during the data transmission phase [38], we showed the training-based lower bound on achievable data rate in Fig. 3. The proposed method also guarantees a good (average) lower bound on achievable rate due to precise channel estimation.

Next, we considered the (more realistic) one-ring channel model which well models typical cellular configurations [14], [16]. The channel spatial correlation with a ULA is given by (3) and depends on AoA $\theta$ and AS $\Delta$, and this model can be extended to the 2-dimensional array case (See [14] for details.) Indeed, we considered a transmitter employing a $10 \times 25$ uniform planar array (UPA) on half-wavelength lattice, $D=1 / 2$ with $N_{r}=1$. In order to compute the vertical and horizontal channel covariance matrices $\mathbf{R}_{V}, \mathbf{R}_{H}$, we assume that the transmit antenna is located at an elevation of $h=60 \mathrm{~m}$, the scattering ring of the receiver has radius $r=30 \mathrm{~m}$, and the distance from the transmitter is $s=100 \mathrm{~m}$. The path loss between the transmitter and the receiver is given by $\gamma=\left(1+\left(s / d_{0}\right)^{\alpha}\right)^{-1}$, where the path loss exponent is set as $\alpha=3.8$ and the reference distance is set as $d_{0}=30 \mathrm{~m}$. Then, the parameters for the channel covariance matrices $\mathbf{R}_{V}$ and $\mathbf{R}_{H}$ are given by $\Delta_{V}=1 / 2(\arctan ((s+r) / h)-\arctan ((s-r) / h))$, $\theta_{V}=1 / 2(\arctan ((s+r) / h)+\arctan ((s-r) / h))$, $\Delta_{H}=\arctan (r / s)$, and $\theta_{H}=\pi / 6$. Finally, the channel

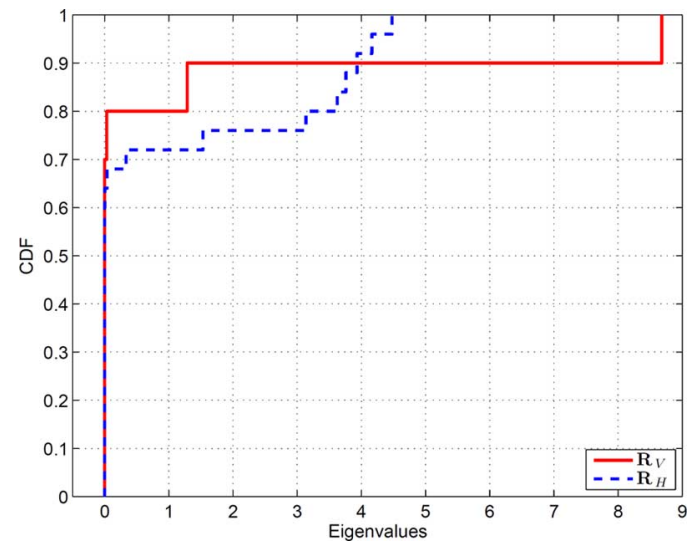

Fig. 4. Empirical eigenvalue CDF of $\mathbf{R}_{V}$ and $\mathbf{R}_{H}$.

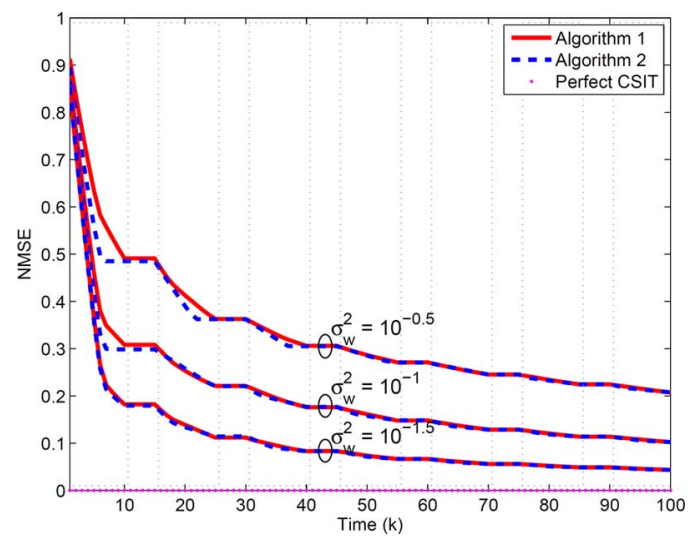

Fig. 5. NMSE versus time index $k$ where $M=15, M_{p}=10$, and $v=$ $3 \mathrm{~km} / \mathrm{h}$.

covariance matrix is given by $\mathbf{R}_{\mathbf{h}}=\mathbf{R}_{H} \otimes \mathbf{R}_{V}$ [16]. Fig. 4 shows the empirical cumulative distribution function (CDF) of the eigenvalues of $\mathbf{R}_{\mathbf{h}}$ obtained in the above, and exhibits rank-deficiency in the spatial channel covariance matrices due to local scattering around the receiver. Note that $70 \%$ to $80 \%$ of the eigenvalues are zero.

Fig. 5 shows the performance of the two proposed algorithms for the considered one-ring channel model: one with fixed pilot power and the other with pilot power design. It is seen that proper power allocation can enhance the channel estimation performance especially both in low SNR and initial tracking periods, but the performance gain is small and the two methods yield almost the same performance at the steady state. Thus, simpler Algorithm 1 with fixed pilot power can be used without much performance loss.

Fig. 6 shows the channel estimation performance of several pilot pattern design methods for the considered one-ring model. It is seen that the proposed method (Algorithm 1) significantly outperforms other pilot design methods both in the transient and steady-state behaviors. Especially, the proposed method yields a received SNR loss of approximately $3 \mathrm{~dB}$ compared to the perfect channel state information case during the transient tracking phase. Orthogonal and random pilot beam patterns are ineffective since they span all the $N_{t}$-dimensional space and such patterns cannot capture the dominant channel uncertainty in space at each pilot symbol time [1]. The fixed $M_{p}$ eigen-direction method outperforms the random or orthogonal pilot 

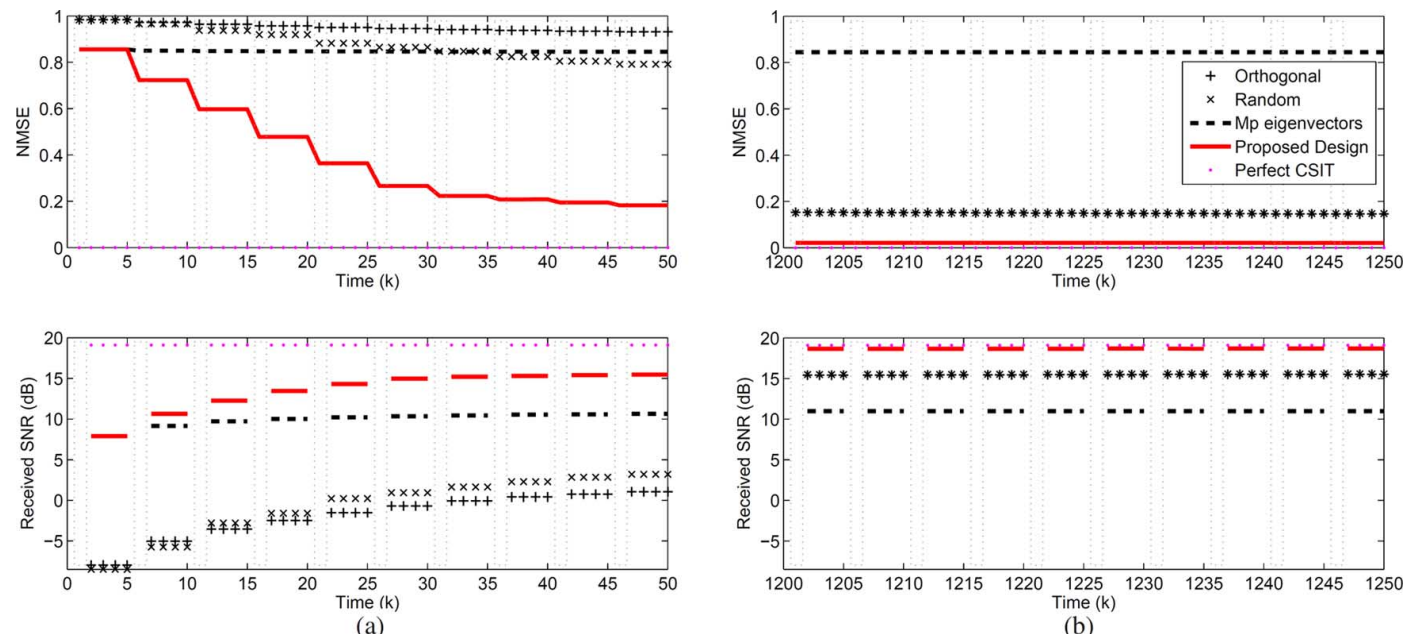

(a)

(b)

Fig. 6. NMSE and received SNR versus time index $k$ where $M=5, M_{p}=1, \sigma_{w}^{2}=10^{-1.5}$, and $v=3 \mathrm{~km} / \mathrm{h}$ (a) Transient tracking (b) Steady-state tracking.

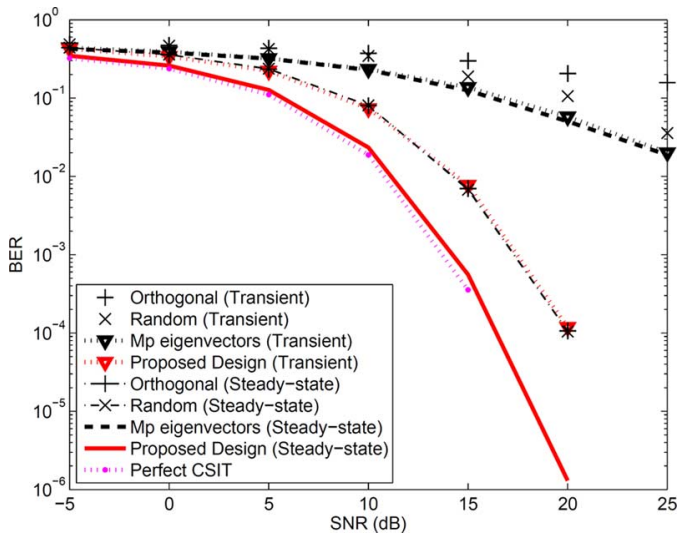

Fig. 7. BER performance for 16-QAM modulation.

design methods in the beginning. This is because the estimated channel from the fixed $M_{p}$ eigen-direction pilot design is a linear combination of the fixed $M_{p}$ eigen-directions, and the use of this channel estimate as the beamforming direction yields a rough channel matching in the beginning. However, as time goes, the channel estimation in the limited subspace is not enough for accurate channel estimation, and this yields the performance saturation. To assess the actual system performance loss due to channel estimation error, we investigated the bit error rate (BER) performance. In Fig. 7, we examine the BER performance based on the estimated channel corresponding to Fig. 6 for the same setup. It is seen that the proposed method significantly outperforms other methods. Note that the channel MSE performance directly affects on the BER performance.

We also investigated the performance variation due to the mobile speed. Fig. 8 shows the steady-state performance of several pilot beam pattern design methods and the corresponding Kalman filtering channel estimation channel as the mobile velocity $v$ varies from $0 \mathrm{~km} / \mathrm{h}$ to $30 \mathrm{~km} / \mathrm{h}$. Note that the proposed design yields much better performance in the case of fast-fading when compared to the other design methods.

Finally, we evaluated the proposed design in the considered one-ring model using the $\mathbf{R}_{t}$ estimation method based on the DFT matrix and the Toeplitz distribution theorem (TDT) presented in Section IV. Fig. 9 shows the received SNR perfor- mance. (Here, we used the block-fading channel Gauss-Markov model in Section III-C since this case was not covered so far, but the performance is not much different from the same for the symbol fading case.) We assumed that AoA and $\Delta$ are known. It is seen that the DFT/TDT-based method yields almost the same performance as the proposed algorithm with perfectly known $\mathbf{R}_{\mathbf{h}}$ ! Thus, the simple practical estimation of $\mathbf{R}_{\mathbf{h}}$ based on the DFT and the TDT seems to work well. Here, to overcome the drawback of the method of using the fixed $M_{p}$ dominant eigenvectors of $\mathbf{R}_{\mathbf{h}}$, we also considered a modified method that initially chooses $L_{p}\left(>M_{p}\right)$ dominant eigenvectors of $250 \times$ $250 \mathbf{R}_{\mathbf{h}}$ and uses $M_{p}$ patterns out of the chosen $L_{p}$ patterns in a round-robin manner. $L_{p}=50$ was used for Fig. 9. Note that up to the first 5 slots the modified method almost tracks the proposed method. This means that roughly 10 eigen-directions out of $L_{p}=50$ are most significant and contain most of the channel power. Hence, if $L_{p}$ were 10, the performance of the modified method should be very good and be comparable to that of the proposed method. However, the problem is that one does not know the number of dominant eigen-directions containing most of the channel power a priori with a proper threshold level. One can view that the proposed algorithm exploits both the most significant eigen-direction and the channel power of each direction over time.

\section{CONCLUSIONS}

We have considered the problem of pilot beam pattern design for massive MIMO systems, and proposed a new method for pilot beam pattern design for massive MIMO systems, based on the stationary Gauss-Markov channel model, by exploiting channel statistics such as temporal and spatial channel correlation that can be used for better system performance. The proposed method yields a greedy (i.e., sequentially optimal) sequence of pilot beam patterns with low computational complexity by exploiting the properties of the Kalman filtering and prediction error covariance matrices. Furthermore, we have considered the joint design problem of pilot beam pattern and pilot beam power and the extension of the proposed method to the case of the block Gauss-Markov channel model. Numerical results have validated the effectiveness of the proposed algo- 


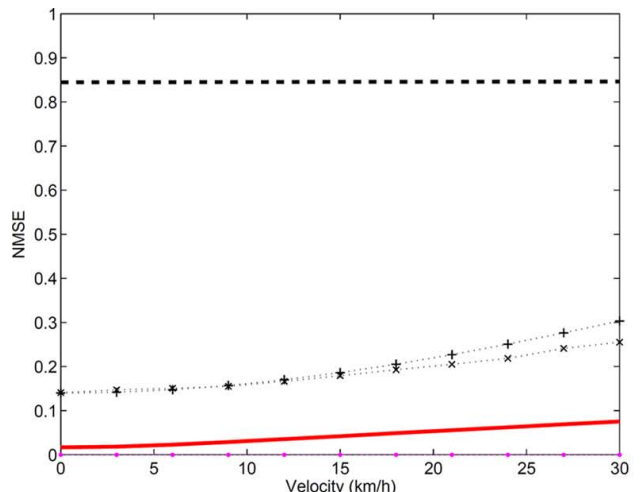

(a)

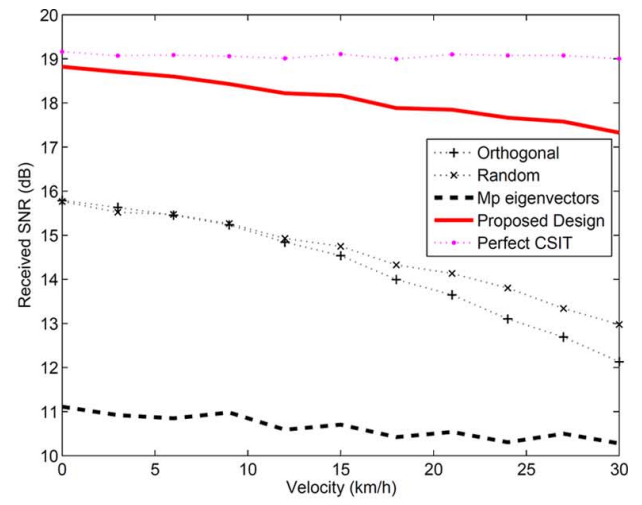

(b)

Fig. 8. NMSE and SNR versus the terminal velocity $v$ where $M=2, M_{p}=1$, and $\sigma_{w}^{2}=10^{-1.5}$ (a) Channel estimation (b) Received SNR.

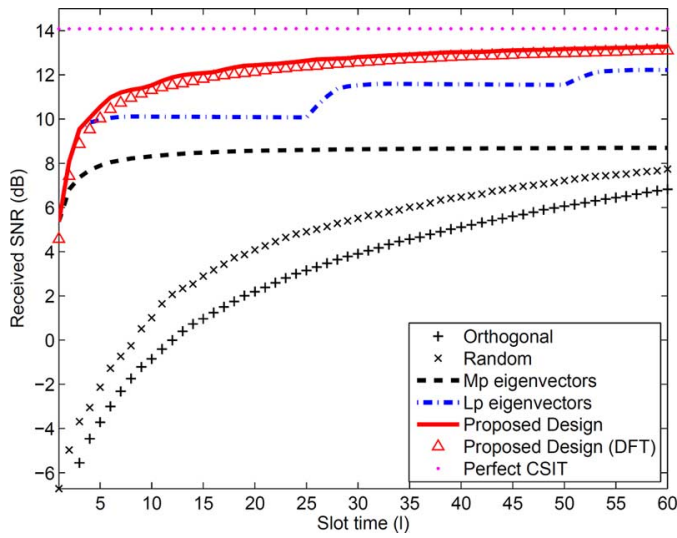

Fig. 9. Received SNR versus slot index $l$ where $M=5, M_{p}=2, \sigma_{w}^{2}=$ $10^{-1}$, and $v=3 \mathrm{~km} / \mathrm{h}$.

rithm, and it is shown that the proposed pilot design method significantly outperforms other pilot design methods especially under the realistic one-ring channel correlation model.

\section{APPENDIX}

\section{Proof of Proposition 1:}

See [1] for the MISO case. We here prove the MIMO case.

Case 1) $k \neq l M+1$ : From (10), $\arg \min _{\mathbf{s}_{k}} \operatorname{tr}\left(\mathbf{P}_{k \mid k}\right)$ can be written as

$\arg \max \operatorname{tr}\left(\mathbf{P}_{k \mid k-1} \mathbf{S}_{k}\left(\mathbf{S}_{k}^{H} \mathbf{P}_{k \mid k-1} \mathbf{S}_{k}+\sigma_{w}^{2} \mathbf{I}_{N_{r}}\right)^{-1} \mathbf{S}_{k}^{H} \mathbf{P}_{k \mid k-1}\right)$.

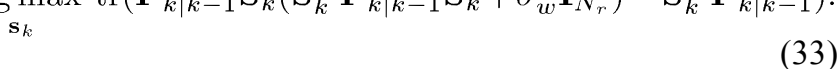

Since $\operatorname{tr}(\mathbf{A B C})=\operatorname{tr}(\mathbf{B C A})$ and $\mathbf{S}_{k}=\mathbf{s}_{k} \otimes \mathbf{I}_{N_{r}}$, the cost function in (33) can be rewritten as

$$
\begin{array}{r}
J=\operatorname{tr}\left\{\left(\left(\mathbf{s}_{k} \otimes \mathbf{I}_{N_{r}}\right)^{H} \mathbf{P}_{k \mid k-1}\left(\mathbf{s}_{k} \otimes \mathbf{I}_{N_{r}}\right)+\sigma_{w}^{2} \mathbf{I}_{N_{r}}\right)^{-1}\right. \\
\left.\left(\mathbf{s}_{k} \otimes \mathbf{I}_{N_{r}}\right)^{H} \mathbf{P}_{k \mid k-1}^{2}\left(\mathbf{s}_{k} \otimes \mathbf{I}_{N_{r}}\right)\right\} .
\end{array}
$$

Since the Kalman prediction error covariance matrix $\mathbf{P}_{k \mid k-1}=(\mathbf{U} \otimes \mathbf{V}) \mathbf{\Lambda}(\mathbf{U} \otimes \mathbf{V})^{H}$ by the assumption, where $\boldsymbol{\Lambda}=\operatorname{diag}\left(\boldsymbol{\Lambda}_{1}, \cdots, \boldsymbol{\Lambda}_{N_{t}}\right), \mathbf{U} \in \mathbb{C}^{N_{t} \times N_{t}}$ and $\mathbf{V} \in \mathbb{C}^{N_{r} \times N_{r}}$, and since the columns of $\mathbf{U}=\left[\mathbf{u}_{1}, \cdots, \mathbf{u}_{N_{t}}\right]$ span $\mathbb{C}^{N_{t}}$, we have $\mathbf{s}_{k}=\sum_{i=1}^{N_{t}} c_{i} \mathbf{u}_{i}$, where $\sum_{i}\left|c_{i}\right|^{2}=\rho_{p}$, and (34) can be rewritten as

$$
\begin{aligned}
J= & \operatorname{tr}\left\{\left(\left[\mathbf{U}^{H} \mathbf{s}_{k} \otimes \mathbf{V}^{H}\right]^{H} \boldsymbol{\Lambda}\left[\mathbf{U}^{H} \mathbf{s}_{k} \otimes \mathbf{V}^{H}\right]+\sigma_{w}^{2} \mathbf{I}_{N_{r}}\right)^{-1}\right. \\
& \left.\left(\mathbf{U}^{H} \mathbf{s}_{k} \otimes \mathbf{V}^{H}\right)^{H} \boldsymbol{\Lambda}^{2}\left(\mathbf{U}^{H} \mathbf{s}_{k} \otimes \mathbf{V}^{H}\right)\right\} \\
= & \operatorname{tr}\left\{\left(\left[\left(\sum_{i} c_{i} \mathbf{e}_{i}\right) \otimes \mathbf{V}^{H}\right]^{H} \boldsymbol{\Lambda}\left[\left(\sum_{i} c_{i} \mathbf{e}_{i}\right) \otimes \mathbf{V}^{H}\right]+\sigma_{w}^{2} \mathbf{I}_{N_{r}}\right)^{-1}\right. \\
& \left.\left(\left(\sum_{i} c_{i} \mathbf{e}_{i}\right) \otimes \mathbf{V}^{H}\right)^{H} \boldsymbol{\Lambda}^{2}\left(\left(\sum_{i} c_{i} \mathbf{e}_{i}\right) \otimes \mathbf{V}^{H}\right)\right\} \\
= & \operatorname{tr}\left\{\left(\sum_{i}\left|c_{i}\right|^{2} \boldsymbol{\Lambda}_{i}+\sigma_{w}^{2} \mathbf{I}_{N_{r}}\right)^{-1}\left(\sum_{i}\left|c_{i}\right|^{2} \boldsymbol{\Lambda}_{i}^{2}\right)\right\}
\end{aligned}
$$

where $\mathbf{e}_{i}$ is the $i$-th unit vector, and the last step (36) holds because

$$
\left(\mathbf{e}_{i} \otimes \mathbf{V}^{H}\right)^{H} \boldsymbol{\Lambda}^{p}\left(\mathbf{e}_{j} \otimes \mathbf{V}^{H}\right)=\delta_{i j} \mathbf{V} \boldsymbol{\Lambda}_{i}^{p} \mathbf{V}^{H},
$$

where $p \in\{1,2\}$ and $\delta_{i j}$ is the Kronecker delta. The cost function (36) can be rewritten as

$$
J\left(c_{1}, \cdots, c_{N_{t}}\right)=\sum_{j=1}^{N_{r}} \frac{\sum_{m}\left|c_{m}\right|^{2} \lambda_{m j}^{2}}{\sum_{n}\left|c_{n}\right|^{2} \lambda_{n j}+\sigma_{w}^{2}},
$$

where $\boldsymbol{\Lambda}_{i}=\operatorname{diag}\left(\lambda_{i 1}, \cdots, \lambda_{i N_{r}}\right)$. The Lagrangian of the optimization of (38) is given by

$$
\mathcal{L}=\sum_{j=1}^{N_{r}} \frac{\sum_{m}\left|c_{m}\right|^{2} \lambda_{m j}^{2}}{\sum_{n}\left|c_{n}\right|^{2} \lambda_{n j}+\sigma_{w}^{2}}+\nu\left(\sum_{m}\left|c_{m}\right|^{2}-\rho_{p}\right),
$$

where $\nu$ is a Lagrange dual variable. The Karush-Kuhn-Tucker (KKT) conditions of the optimization of (38) are given by

$$
\begin{aligned}
0= & \frac{\partial \mathcal{L}}{\partial c_{i}^{*}} \\
= & \sum_{j} \frac{c_{i} \lambda_{i j}^{2}\left(\sum_{n}\left|c_{n}\right|^{2} \lambda_{n j}+\sigma_{w}^{2}\right)-c_{i} \lambda_{i j}\left(\sum_{m}\left|c_{m}\right|^{2} \lambda_{m j}^{2}\right)}{\left(\sum_{n}\left|c_{n}\right|^{2} \lambda_{n j}+\sigma_{w}^{2}\right)^{2}} \\
& +\nu c_{i} .
\end{aligned}
$$

It is easy to verify that $c_{i^{\prime}}=\rho_{p} e^{\ell \theta}$ for some $i^{\prime} \in\left\{1,2, \cdots, N_{t}\right\}$ and $c_{i}=0$ for all $i \neq i^{\prime}$ with $\nu=-\sum_{j} \lambda_{i^{\prime} j}^{2} \sigma_{w}^{2} /\left(\rho_{p} \lambda_{i^{\prime} j}^{2}\right)^{2}$ satisfies the KKT conditions. Since (38) is not convex in terms of $\left\{c_{i}\right\}$, the solution to the KKT conditions is not unique. However, all such solutions with only one non-zero $c_{i}$ are stationary points of the optimization, i.e., each of them is a local optimum. 
Among such solutions the best one is given by $c_{i}=\sqrt{\rho_{p}}$ for $i=i_{k}$ and $c_{i}=0$ for all $i \neq i_{k}$, where

$$
\begin{aligned}
i_{k} & :=\arg \max _{i} \operatorname{tr}\left\{\left(\rho_{p} \boldsymbol{\Lambda}_{i}+\sigma_{w}^{2} \mathbf{I}_{N_{r}}\right)^{-1}\left(\rho_{p} \boldsymbol{\Lambda}_{i}^{2}\right)\right\} \\
& =\arg \max _{i} \sum_{j=1}^{N_{r}} \rho_{p} \lambda_{i j}^{2}\left(\rho_{p} \lambda_{i j}+\sigma_{w}^{2}\right)^{-1},
\end{aligned}
$$

and $\mathbf{s}_{k}=\sqrt{\rho_{p}} \mathbf{u}_{i_{k}}$ is a locally optimal solution to minimizing $\operatorname{tr}\left(\mathbf{P}_{k \mid k}\right)$.

Case 2) $k=l M+1$ : We have $M_{d}$ prediction steps without a measurement update step before the first pilot symbol time $k$ in the $l$-th slot. In this case, still the measurement update form (10) at $k$ is valid with $\mathbf{P}_{k \mid k-1}$ replaced by the error covariance matrix $\mathbf{P}_{k \mid(l-1) M+M_{p}}$ of the Kalman prediction for time $k$ based on all the previous pilot beam patterns. Hence, the proof in Case 1) is applicable to this case just with $\mathbf{P}_{k \mid k-1}$ replaced by $\mathbf{P}_{k \mid(l-1) M+M_{p}}$.

Derivation of $\mathbf{P}_{k \mid K}$ :

$$
\begin{aligned}
& \mathbf{P}_{k \mid k} \\
= & \mathbf{P}_{k \mid k-1}-\mathbf{P}_{k \mid k-1} \mathbf{S}_{k}\left(\mathbf{S}_{k}^{H} \mathbf{P}_{k \mid k-1} \mathbf{S}_{k}+\sigma_{w}^{2} \mathbf{I}_{N_{r}}\right)^{-1} \mathbf{S}_{k}^{H} \mathbf{P}_{k \mid k-1} \\
= & (\mathbf{U} \otimes \mathbf{V}) \boldsymbol{\Lambda}^{(k)}(\mathbf{U} \otimes \mathbf{V})^{H}-(\mathbf{U} \otimes \mathbf{V}) \boldsymbol{\Lambda}^{(k)}\left(\sqrt{\rho_{p}} \mathbf{e}_{i_{k}} \otimes \mathbf{V}^{H}\right) \\
& {\left[\left(\sqrt{\rho_{p}} \mathbf{e}_{i_{k}} \otimes \mathbf{V}^{H}\right)^{H} \boldsymbol{\Lambda}^{(k)}\left(\sqrt{\rho_{p}} \mathbf{e}_{i_{k}} \otimes \mathbf{V}^{H}\right)+\sigma_{w}^{2} \mathbf{I}_{N_{r}}\right]^{-1} } \\
& \left(\sqrt{\rho_{p}} \mathbf{e}_{i_{k}} \otimes \mathbf{V}^{H}\right)^{H} \boldsymbol{\Lambda}^{(k)}(\mathbf{U} \otimes \mathbf{V})^{H} \\
\stackrel{(a)}{=} & (\mathbf{U} \otimes \mathbf{V}) \boldsymbol{\Lambda}^{(k)}(\mathbf{U} \otimes \mathbf{V})^{H}-(\mathbf{U} \otimes \mathbf{V}) \boldsymbol{\Lambda}^{(k)}\left(\sqrt{\rho_{p}} \mathbf{e}_{i_{k}} \otimes \mathbf{V}^{H}\right) \\
& {\left[\mathbf{V}\left(\rho_{p} \boldsymbol{\Lambda}_{i_{k}}^{(k)}+\sigma_{w}^{2} \mathbf{I}_{N_{r}}\right)^{-1} \mathbf{V}^{H}\right]\left(\sqrt{\rho_{p}} \mathbf{e}_{i_{k}} \otimes \mathbf{V}^{H}\right)^{H} } \\
& \boldsymbol{\Lambda}^{(k)}(\mathbf{U} \otimes \mathbf{V})^{H} \\
\stackrel{(b)}{=} & (\mathbf{U} \otimes \mathbf{V}) \boldsymbol{\Lambda}^{(k)}(\mathbf{U} \otimes \mathbf{V})^{H}-(\mathbf{U} \otimes \mathbf{V}) \boldsymbol{\Lambda}^{(k)} \\
& {\left[\rho_{p}\left(\mathbf{e}_{i_{k}} \mathbf{e}_{i_{k}}^{T}\right) \otimes\left(\rho_{p} \boldsymbol{\Lambda}_{i_{k}}^{(k)}+\sigma_{w}^{2} \mathbf{I}_{N_{r}}\right)^{-1}\right] \boldsymbol{\Lambda}^{(k)}(\mathbf{U} \otimes \mathbf{V})^{H} } \\
\stackrel{(c)}{=} & (\mathbf{U} \otimes \mathbf{V}) \boldsymbol{\Lambda}^{(k)}(\mathbf{U} \otimes \mathbf{V})^{H}-(\mathbf{U} \otimes \mathbf{V})\left\{\left(\mathbf{e}_{i_{k}} \mathbf{e}_{i_{k}}^{T}\right) \otimes\right. \\
& {\left.\left[\rho_{p} \boldsymbol{\Lambda}_{i_{k}}^{(k)}\left(\rho_{p} \boldsymbol{\Lambda}_{i_{k}}^{(k)}+\sigma_{w}^{2} \mathbf{I}_{N_{r}}\right)^{-1} \boldsymbol{\Lambda}_{i_{k}}^{(k)}\right]\right\}(\mathbf{U} \otimes \mathbf{V})^{H} } \\
= & (\mathbf{U} \otimes \mathbf{V})\left\{\mathbf{\Lambda}^{(k)}-\left(\mathbf{e}_{i_{k}} \mathbf{e}_{i_{k}}^{T}\right) \otimes\right. \\
& {\left.\left[\rho_{p} \boldsymbol{\Lambda}_{i_{k}}^{(k)}\left(\rho_{p} \boldsymbol{\Lambda}_{i_{k}}^{(k)}+\sigma_{w}^{2} \mathbf{I}_{N_{r}}\right)^{-1} \boldsymbol{\Lambda}_{i_{k}}^{(k)}\right]\right\}(\mathbf{U} \otimes \mathbf{V})^{H}, }
\end{aligned}
$$

where the equality $(a)$ follows because

$$
\begin{array}{r}
{\left[\left(\sqrt{\rho_{p}} \mathbf{e}_{i_{k}} \otimes \mathbf{V}^{H}\right)^{H} \boldsymbol{\Lambda}^{(k)}\left(\sqrt{\rho_{p}} \mathbf{e}_{i_{k}} \otimes \mathbf{V}^{H}\right)+\sigma_{w}^{2} \mathbf{I}_{N_{r}}\right]^{-1}} \\
=\mathbf{V}\left(\rho_{p} \boldsymbol{\Lambda}_{i_{k}}^{(k)}+\sigma_{w}^{2} \mathbf{I}_{N_{r}}\right)^{-1} \mathbf{V}^{H}
\end{array}
$$

and the equality $(b)$ follows because $\left(\mathbf{A}_{1} \mathbf{A}_{2}\right) \otimes\left(\mathbf{B}_{1} \mathbf{B}_{2}\right)=$ $\left(\mathbf{A}_{1} \otimes \mathbf{B}_{1}\right)\left(\mathbf{A}_{2} \otimes \mathbf{B}_{2}\right)$. The equality $(c)$ holds because

$$
\begin{aligned}
\boldsymbol{\Lambda}^{(k)}\left[\rho_{p}\left(\mathbf{e}_{i_{k}} \mathbf{e}_{i_{k}}^{T}\right) \otimes\left(\rho_{p} \boldsymbol{\Lambda}_{i_{k}}^{(k)}+\sigma_{w}^{2} \mathbf{I}_{N_{r}}\right)^{-1}\right] \boldsymbol{\Lambda}^{(k)} \\
=\left(\mathbf{e}_{i_{k}} \mathbf{e}_{i_{k}}^{T}\right) \otimes\left[\rho_{p} \boldsymbol{\Lambda}_{i_{k}}^{(k)}\left(\rho_{p} \boldsymbol{\Lambda}_{i_{k}}^{(k)}+\sigma_{w}^{2} \mathbf{I}_{N_{r}}\right)^{-1} \boldsymbol{\Lambda}_{i_{k}}^{(k)}\right] .
\end{aligned}
$$

\section{Proof of Proposition 3:}

For the $l$-th pilot transmission period $(k=l M+m)$, let $\boldsymbol{\rho}=$ $\left[\rho_{l M+1}, \ldots, \rho_{l M+M_{p}}\right]^{T}$ be a power allocation vector with the pilot beam pattern sequence determined by $\left\{\mathcal{K}_{i}, 1 \leq i \leq N_{t}\right\}$. The MSE at time $l M+M_{p}$ is given by

$$
\begin{aligned}
& \operatorname{tr}\left(\mathbf{P}_{\left.l M+M_{p} \mid l M+M_{p}\right)}\right) \\
= & \sum_{i=1}^{N_{t}} \operatorname{tr}\left(\overline{\boldsymbol{\Lambda}}_{i}^{\left(l M+M_{p}\right)}\right) \\
= & \sum_{i=1}^{N_{t}} \operatorname{tr}\left(a^{2\left(\bar{p}_{i}-1\right)} \bar{\Lambda}_{i}^{\left(k_{\left|\mathcal{K}_{i}\right|}^{i}\right)}+\left(1-a^{2\left(\bar{p}_{i}-1\right)}\right) \boldsymbol{\Lambda}_{i}^{(1)}\right),
\end{aligned}
$$

where $\overline{\boldsymbol{\Lambda}}_{i}^{(k)} \in \mathbb{R}^{N_{r} \times N_{r}}$ is the $i$-th diagonal sub-block of $\overline{\boldsymbol{\Lambda}}^{(k)}$ defined (20). $\left(\bar{p}_{i}=M_{p}+1, k_{\left|\mathcal{K}_{i}\right|}^{i}=l M\right.$ when $\left.\left|\mathcal{K}_{i}\right|=0.\right)(42)$ holds because $\mathbf{u}_{i}$ only affects the $i$-th subblock of the eigenvalue matrix and the MSE for the $i$-th block at the end of the pilot period is given by channel prediction from the last pilot use of $\mathbf{u}_{i}$ at time $k_{\left|\mathcal{K}_{i}\right|}^{i}$. Combining Kalman prediction and measurement update steps, we have

$$
\begin{aligned}
& \operatorname{tr}\left(\overline{\boldsymbol{\Lambda}}_{i}^{\left(k_{j}^{i}\right)}\right) \\
= & \operatorname{tr}\left(\frac{\sigma_{w}^{2}\left(a^{2 p_{j}^{i}} \overline{\boldsymbol{\Lambda}}_{i}^{\left(k_{j-1}^{i}\right)}+\left(1-a^{2 p_{j}^{i}}\right) \boldsymbol{\Lambda}_{i}^{(1)}\right)}{\rho_{k_{j}^{i}}\left(a^{2 p_{j}^{i}} \overline{\boldsymbol{\Lambda}}_{i}^{\left(k_{j-1}^{i}\right)}+\left(1-a^{2 p_{j}^{i}}\right) \boldsymbol{\Lambda}_{i}^{(1)}\right)+\sigma_{w}^{2} \mathbf{I}_{N_{r}}}\right) \\
= & f\left(\overline{\boldsymbol{\Lambda}}_{i, 0}^{\left(k_{j-1}^{i}\right)}\right)
\end{aligned}
$$

where $k_{j}^{i} \in \mathcal{K}_{i}, f\left(\overline{\boldsymbol{\Lambda}}_{i, \epsilon}^{\left(k_{j-1}^{i}\right)}\right)$, and $\overline{\boldsymbol{\Lambda}}_{i, \epsilon}^{\left(k_{j-1}^{i}\right)}$ are defined in (46), (47), respectively. (Here, we have slight abuse of notation. $\mathbf{A} / \mathbf{B}$ means $\mathbf{B}^{-1} \mathbf{A}$ for two matrices $\mathbf{A}$ and $\mathbf{B}$.) Proof is by an iterative argument. We start from $j=\left|\mathcal{K}_{i}\right|$ and $j-1=\left|\mathcal{K}_{i}\right|-1$ for the original $\mathcal{K}_{i}$. By Lemma 1 and Remark 1, (43) is reduced by updating $\tilde{\rho}_{k_{j}^{i}}=\rho_{k_{j}^{i}}+\rho_{k_{j-1}^{i}}$ and $\tilde{\rho}_{k_{j-1}^{i}}=0$, when we consider the two power values for $j-1$ and $j$. With this improvement, we construct a new $\mathcal{K}_{i}^{\prime}=\left\{k_{1}^{i}, \cdots, k_{j-2}^{i}, k_{\left|\mathcal{K}_{i}\right|}^{i}\right\}$ with $\left|\mathcal{K}_{i}\right|=\left|\mathcal{K}_{i}\right|-1$ and a new power allocation $\left[\rho_{k_{1}^{i}}, \cdots, \rho_{k_{\left|\mathcal{K}_{i}\right|-2}}, \rho_{k_{\left|\mathcal{K}_{i}\right|-1}^{i}}+\rho_{k_{\left|\mathcal{K}_{i}\right|}^{i}}\right]^{T}$ for $\mathcal{K}_{i}^{\prime}$. Then, we apply the same argument to the last two power terms of the newly constructed $\mathcal{K}_{i}^{\prime}$. In this way, (43) is minimized by allocating all the power for the $i$-th eigen-direction to $k_{\left|\mathcal{K}_{i}\right|}^{i}$ for the original $\mathcal{K}_{i}$. Since (42) is a monotone increasing function of $\operatorname{tr}\left(\overline{\boldsymbol{\Lambda}}_{i}^{\left(k_{j}^{i}\right)}\right)$, we have the claim.

Lemma 1: Given any $\rho_{k_{j-1}^{i}}, \rho_{k_{j}^{i}} \in \mathbb{R}_{+}$, set $\tilde{\rho}_{k_{j-1}^{i}}=\rho_{k_{j-1}^{i}}-\epsilon$ and $\tilde{\rho}_{k_{j}^{i}}=\rho_{k_{j}^{i}}+\epsilon$ for any $\epsilon \in\left[0, \rho_{k_{j-1}^{i}}\right]$. Then, the following holds:

$$
f\left(\overline{\mathbf{\Lambda}}_{i, 0}^{\left(k_{j-1}^{i}\right)}\right)-f\left(\overline{\boldsymbol{\Lambda}}_{i, \epsilon}^{\left(k_{j-1}^{i}\right)}\right) \geq 0
$$

where

$$
\begin{aligned}
& f\left(\overline{\boldsymbol{\Lambda}}_{i, \epsilon}^{\left(k_{j-1}^{i}\right)}\right)=\operatorname{tr}\left\{\left(\sigma_{w}^{2}\left(a^{2 p_{j}^{i}} \overline{\boldsymbol{\Lambda}}_{i, \epsilon}^{\left(k_{j-1}^{i}\right)}+\left(1-a^{2 p_{j}^{i}}\right) \boldsymbol{\Lambda}_{i}^{(1)}\right)\right)\right. \\
& \left(\tilde{\rho}_{k_{j}^{i}}\left(a^{2 p_{j}^{i}} \overline{\boldsymbol{\Lambda}}_{i, \epsilon}^{\left(k_{j-1}^{i}\right)}+\left(1-a^{2 p_{j}^{i}}\right) \boldsymbol{\Lambda}_{i}^{(1)}\right)\right. \\
& \left.\left.+\sigma_{w}^{2} \mathbf{I}_{N_{r}}\right)^{-1}\right\} \\
& \overline{\boldsymbol{\Lambda}}_{i, \epsilon}^{\left(k_{j-1}^{i}\right)}=\sigma_{w}^{2} \boldsymbol{\Lambda}_{i}^{\left(k_{j-1}^{i}\right)}\left(\tilde{\rho}_{k_{j-1}^{i}} \boldsymbol{\Lambda}_{i}^{\left(k_{j-1}^{i}\right)}+\sigma_{w}^{2} \mathbf{I}_{N_{r}}\right)^{-1} \\
& \boldsymbol{\Lambda}_{i}^{\left(k_{j-1}^{i}\right)}=a^{2 p_{j-1}^{i}} \overline{\boldsymbol{\Lambda}}_{i}^{\left(k_{j-2}^{i}\right)}+\left(1-a^{2 p_{j-1}^{i}}\right) \boldsymbol{\Lambda}_{i}^{(1)} \\
& k_{0}^{i}=l M, k_{j}^{i} \in \mathcal{K}_{i} \text {, and } 2 \leq j \leq\left|\mathcal{K}_{i}\right| .
\end{aligned}
$$

Proof: For notational simplicity, we omit the upper index $i$ of $k_{j}^{i}$ and $p_{j}^{i}$ when there is no ambiguity. De- 
fine $\tilde{\mathbf{D}}:=a^{2 p_{j}} \overline{\boldsymbol{\Lambda}}_{i, \epsilon}^{\left(k_{j-1}\right)}+\left(1-a^{2 p_{j}}\right) \boldsymbol{\Lambda}_{i}^{(1)}$ and $\mathbf{D}:=$ $a^{2 p_{j}} \overline{\boldsymbol{\Lambda}}_{i}^{\left(k_{j-1}\right)}+\left(1-a^{2 p_{j}}\right) \boldsymbol{\Lambda}_{i}^{(1)}$ with $\overline{\boldsymbol{\Lambda}}_{i}^{\left(k_{j-1}\right)}:=\left.\overline{\boldsymbol{\Lambda}}_{i, \epsilon}^{\left(k_{j-1}\right)}\right|_{\epsilon=0}$. Then, (45) can be rewritten as

$$
\begin{aligned}
& \operatorname{tr}\left(\frac{\sigma_{w}^{2} \mathbf{D}}{\rho_{k_{j}} \mathbf{D}+\sigma_{w}^{2} \mathbf{I}_{N_{r}}}-\frac{\sigma_{w}^{2} \tilde{\mathbf{D}}}{\tilde{\rho}_{k_{j}} \tilde{\mathbf{D}}+\sigma_{w}^{2} \mathbf{I}_{N_{r}}}\right) \\
& =\operatorname{tr}\left(\frac{\sigma_{w}^{2}\left(\epsilon \mathbf{D} \tilde{\mathbf{D}}+\sigma_{w}^{2}(\mathbf{D}-\tilde{\mathbf{D}})\right)}{\left(\rho_{k_{j}} \mathbf{D}+\sigma_{w}^{2} \mathbf{I}_{N_{r}}\right)\left(\tilde{\rho}_{k_{j}} \tilde{\mathbf{D}}+\sigma_{w}^{2} \mathbf{I}_{N_{r}}\right)}\right) .
\end{aligned}
$$

Note that the denominator of the right-hand side (RHS) in (50) is obviously positive definite and the numerator is also positive semi-definite because each term on the RHS in (51) is positive semi-definite because

$$
\begin{aligned}
& \epsilon \mathbf{D} \tilde{\mathbf{D}}+\sigma_{w}^{2}(\mathbf{D}-\tilde{\mathbf{D}}) \\
= & \epsilon\left(1-a^{2 p_{j}}\right)^{2}\left(\boldsymbol{\Lambda}_{i}^{(1)}\right)^{2}+\epsilon \frac{a^{2 p_{j}}\left(1-a^{2 p_{j}}\right) \sigma_{w}^{2} \boldsymbol{\Lambda}_{i}^{\left(k_{j-1}\right)}}{\left(\rho_{k_{j}} \mathbf{D}+\sigma_{w}^{2} \mathbf{I}_{N_{r}}\right)\left(\tilde{\rho}_{k_{j}} \tilde{\mathbf{D}}+\sigma_{w}^{2} \mathbf{I}_{N_{r}}\right)} \\
& {\left[\left(2 \rho_{k_{j-1}}-\epsilon\right) \boldsymbol{\Lambda}_{i}^{(1)} \boldsymbol{\Lambda}_{i}^{\left(k_{j-1}\right)}+\right.} \\
& \left.\sigma_{w}^{2}\left(\boldsymbol{\Lambda}_{i}^{(1)}+a^{2 p_{j-1}}\left(\boldsymbol{\Lambda}_{i}^{(1)}-\overline{\boldsymbol{\Lambda}}_{i}^{\left(k_{j-2}\right)}\right)\right)\right] .
\end{aligned}
$$

Note that $\Lambda_{i}^{(1)} \succeq \bar{\Lambda}_{i}^{(k)}$ for all $k$. (Remember that the channel is stationary and the measurement update only improves the channel estimation quality.) Hence, we have the claim.

Remark 1: In case that we control $\rho_{k_{j-1}}, \rho_{k_{j}} \in \mathbb{R}_{+}$, $f\left(\bar{\Lambda}_{i, \epsilon}^{\left(k_{j-1}\right)}\right)$ is minimized when $\epsilon=\epsilon^{\prime}:=\rho_{k_{j-1}}$. This can easily be shown by $f\left(\bar{\Lambda}_{i, \epsilon}^{\left(k_{j-1}\right)}\right)-f\left(\overline{\boldsymbol{\Lambda}}_{i, \epsilon^{\prime}}^{\left(k_{j-1}\right)}\right) \geq 0$. One can write a similar equation to (50). Although the detail is not shown here, in this case the corresponding denominator is positive definite and the corresponding numerator includes obviously positive semi-definite term and the term

$$
\begin{aligned}
& \left(\rho_{k_{j-1}}-\epsilon\right)\left(1-a^{2 p_{j}}\right)\left[\boldsymbol { \Lambda } _ { i } ^ { ( 1 ) } \left(\left(1-a^{2\left(p_{j-1}+p_{j}\right)}\right) \boldsymbol{\Lambda}_{i}^{(1)}+\right.\right. \\
& \left.\left.a^{2\left(p_{j-1}+p_{j}\right)} \overline{\boldsymbol{\Lambda}}_{i}^{\left(k_{j}\right)}\right)+a^{2\left(p_{j-1}+p_{j}\right)}\left(\boldsymbol{\Lambda}_{i}^{(1)}-\overline{\boldsymbol{\Lambda}}_{i}^{\left(k_{j}\right)}\right) \overline{\boldsymbol{\Lambda}}_{i, \epsilon}^{\left(k_{j-1}\right)}\right],
\end{aligned}
$$

which is positive semi-definite.

\section{Proof of Proposition 4:}

From (10) and (34), $\arg \min _{\mathbf{S}_{l}} \operatorname{tr}\left(\mathbf{P}_{l \mid l}\right)$ can be written as

$$
\underset{\mathbf{S}_{l}}{\arg \max } \operatorname{tr}\left(\left[\mathbf{S}_{l}^{H} \mathbf{P}_{l \mid l-1} \mathbf{S}_{l}+\sigma_{w}^{2} \mathbf{I}_{M_{p}}\right]^{-1} \mathbf{S}_{l}^{H} \mathbf{P}_{l \mid l-1}^{2} \mathbf{S}_{l}\right) \text {. }
$$

For orthogonal pilot signals, the objective function (52) can be rewritten as

$$
\operatorname{tr}\left(\left[\mathbf{S}_{l}^{H}\left(\mathbf{P}_{l \mid l-1}+\frac{\sigma_{w}^{2}}{\rho_{p}} \mathbf{I}_{N_{t}}\right) \mathbf{S}_{l}\right]^{-1} \mathbf{S}_{l}^{H} \mathbf{P}_{l \mid l-1}^{2} \mathbf{S}_{l}\right) .
$$

Define $\mathbf{P}_{l, \sigma_{w}}:=\mathbf{P}_{l \mid l-1}+\sigma_{w}^{2} / \rho_{p} \mathbf{I}_{N_{t}}=\mathbf{P}_{l, \sigma_{w}}^{1 / 2} \mathbf{P}_{l, \sigma_{w}}^{H / 2}$ and $\mathbf{F}:=$ $\mathbf{P}_{l, \sigma_{w}}^{H / 2} \mathbf{S}_{l}$. Then, (53) can be rewritten as

$$
\begin{aligned}
& \operatorname{tr}\left(\left(\mathbf{F}^{H} \mathbf{F}\right)^{-1} \mathbf{F}^{H} \mathbf{P}_{l, \sigma_{w}}^{-1 / 2} \mathbf{P}_{l \mid l-1}^{2} \mathbf{P}_{l, \sigma_{w}}^{-H / 2} \mathbf{F}\right) \\
\stackrel{(a)}{=} & \operatorname{tr}\left(\left(\mathbf{F}^{H} \mathbf{F}\right)^{-H / 2} \mathbf{F}^{H} \mathbf{P}_{l, \sigma_{w}}^{-1 / 2} \mathbf{P}_{l \mid l-1}^{2} \mathbf{P}_{l, \sigma_{w}}^{-H / 2} \mathbf{F}\left(\mathbf{F}^{H} \mathbf{F}\right)^{-1 / 2}\right) \\
= & \operatorname{tr}\left(\mathbf{B}^{H} \mathbf{P}_{l, \sigma_{w}}^{-1 / 2} \mathbf{P}_{l \mid l-1}^{2} \mathbf{P}_{l, \sigma_{w}}^{-H / 2} \mathbf{B}\right),
\end{aligned}
$$

where $\mathbf{B}:=\mathbf{F}\left(\mathbf{F}^{H} \mathbf{F}\right)^{-1 / 2}$. The equality $(a)$ holds by the positive definiteness of $\mathbf{F}^{H} \mathbf{F}$ and $\operatorname{tr}(\mathbf{A B C})=\operatorname{tr}(\mathbf{B C A})$. Because $\mathbf{B}^{H} \mathbf{B}=\mathbf{I}_{M_{p}}$, the optimal $\mathbf{B}$ that maximizes (54) is given by the $M_{p}$ dominant eigenvectors of $\mathbf{P}_{l, \sigma_{w}}^{-1 / 2} \mathbf{P}_{l \mid l-1}^{2} \mathbf{P}_{l, \sigma_{w}}^{-H / 2}$ by Ky-Fan
[51]. Let the ED of $\mathbf{P}_{l \mid l-1}$ be $\mathbf{P}_{l \mid l-1}=\mathbf{U} \boldsymbol{\Lambda}^{(l)} \mathbf{U}^{H}$, where the diagonal matrix $\boldsymbol{\Lambda}^{(l)}$ contains the eigenvalues of $\mathbf{P}_{l \mid l-1}$ in a decreasing order. Then, $\mathbf{P}_{l, \sigma_{w}}^{-1 / 2} \mathbf{P}_{l \mid l-1}^{2} \mathbf{P}_{l, \sigma_{w}}^{-H / 2}$ is given by

$$
\mathbf{P}_{l, \sigma_{w}}^{-1 / 2} \mathbf{P}_{l \mid l-1}^{2} \mathbf{P}_{l, \sigma_{w}}^{-H / 2}=\mathbf{U}\left(\frac{\left(\boldsymbol{\Lambda}^{(l)}\right)^{2}}{\Lambda^{(l)}+\frac{\sigma_{w}^{2}}{\rho_{p}} \mathbf{I}_{N_{t}}}\right) \mathbf{U}^{H}
$$

from $\mathbf{P}_{l, \sigma_{w}}=\mathbf{U}\left(\boldsymbol{\Lambda}^{(l)}+\sigma_{w}^{2} / \rho_{p} \mathbf{I}_{N_{t}}\right) \mathbf{U}^{H}$. Since $g(x)=$ $x^{2} /\left(x+\sigma^{2}\right), x \geq 0$ is a monotone increasing function of $x, \mathbf{B}=\mathbf{U}\left(:, 1: M_{p}\right)$ which is achieved by $\mathbf{S}_{l}=\sqrt{\rho_{p}} \mathbf{U}\left(:, 1: M_{p}\right)$.

\section{Power Allocation:}

The problem of (26) can be solved by the standard convex optimization method. The Lagrangian of the problem is given by

$$
\begin{aligned}
L(\boldsymbol{\rho}, \boldsymbol{\xi}, \nu)=\sum_{i:\left|\mathcal{K}_{i}\right|=1} \sum_{j=1}^{N_{r}} \frac{a^{2\left(l M+M_{p}-k^{i}\right)} \sigma_{w}^{2} \lambda_{i j}^{\left(k^{i}\right)}}{\rho_{k^{i}} \lambda_{i j}^{\left(k^{i}\right)}+\sigma_{w}^{2}}- \\
\sum_{i:\left|\mathcal{K}_{i}\right|=1} \xi_{k^{i}} \rho_{k^{i}}+\nu\left(\sum_{i:\left|\mathcal{K}_{i}\right|=1} \rho_{k^{i}}-M_{p} \rho_{p}\right),
\end{aligned}
$$

where $\xi_{k^{i}}$ and $\nu$ are the Lagrange multipliers associated to the constraints, and $\boldsymbol{\lambda}_{i}^{\left(k^{i}\right)}=\operatorname{diag}\left(\boldsymbol{\Lambda}_{i}^{\left(k^{i}\right)}\right)$ for $k^{i} \in \mathcal{K}_{i}$. The KarushKuhn-Tucker (KKT) conditions are then written as

$$
\begin{aligned}
\rho_{k^{i}} & \geq 0, \sum_{i:\left|\mathcal{K}_{i}\right|=1} \rho_{k^{i}}=M_{p} \rho_{p} \\
\xi_{k^{i}} & \geq 0, \xi_{k^{i}} \rho_{k^{i}}=0 \\
\frac{\partial L(\boldsymbol{\rho}, \boldsymbol{\xi}, \nu)}{\partial \rho_{k^{i}}} & =-\sum_{j=1}^{N_{r}} \frac{a^{2\left(l M+M_{p}-k^{i}\right)} \sigma_{w}^{2}\left(\lambda_{i j}^{\left(k^{i}\right)}\right)^{2}}{\left(\rho_{k^{i}} \lambda_{i j}^{\left(k^{i}\right)}+\sigma_{w}^{2}\right)^{2}}-\xi_{k^{i}}+\nu \\
& =0
\end{aligned}
$$

From the above conditions, we have

$$
\begin{array}{r}
\sum_{j=1}^{N_{r}} \frac{a^{2\left(l M+M_{p}-k^{i}\right)} \sigma_{w}^{2}\left(\lambda_{i j}^{\left(k^{i}\right)}\right)^{2}}{\left(\rho_{k^{i}} \lambda_{i j}^{\left(k^{i}\right)}+\sigma_{w}^{2}\right)^{2}} \leq \nu, \\
\left(\nu-\sum_{j=1}^{N_{r}} \frac{a^{2\left(l M+M_{p}-k^{i}\right)} \sigma_{w}^{2}\left(\lambda_{i j}^{\left(k^{i}\right)}\right)^{2}}{\left(\rho_{k^{i}} \lambda_{i j}^{\left(k^{i}\right)}+\sigma_{w}^{2}\right)^{2}}\right) \rho_{k^{i}}=0 .
\end{array}
$$

If $a^{2\left(l M+M_{p}-k^{i}\right)} / \sigma_{w}^{2} \sum_{j=1}^{N_{r}}\left(\lambda_{i j}^{\left(k^{i}\right)}\right)^{2}>\nu$, (58) holds only if $\rho_{k^{i}}>0$, and by (59) this implies that

$$
\nu=\sum_{j=1}^{N_{r}} \frac{a^{2\left(l M+M_{p}-k^{i}\right)} \sigma_{w}^{2}\left(\lambda_{i j}^{\left(k^{i}\right)}\right)^{2}}{\left(\rho_{k^{i}} \lambda_{i j}^{\left(k^{i}\right)}+\sigma_{w}^{2}\right)^{2}} .
$$

If $a^{2\left(l M+M_{p}-k^{i}\right)} / \sigma_{w}^{2} \sum_{j=1}^{N_{r}}\left(\lambda_{i j}^{\left(k^{i}\right)}\right)^{2} \leq \nu$, then $\rho_{k^{i}}=0$ because we have

$\nu \geq \frac{a^{2\left(l M+M_{p}-k^{i}\right)}}{\sigma_{w}^{2}} \sum_{j=1}^{N_{r}}\left(\lambda_{i j}^{\left(k^{i}\right)}\right)^{2}>\sum_{j=1}^{N_{r}} \frac{a^{2\left(l M+M_{p}-k^{i}\right)} \sigma_{w}^{2}\left(\lambda_{i j}^{\left(k^{i}\right)}\right)^{2}}{\left(\rho_{k^{i}} \lambda_{i j}^{\left(k^{i}\right)}+\sigma_{w}^{2}\right)^{2}}$

(59) holds only if $\rho_{k^{i}}=0$. 
When $N_{r}=1$, the optimal power allocation is determined from $(60)$ as

$$
\rho_{k^{i}}=\left(a^{l M+M_{p}-k^{i}} \sigma_{w} \nu^{-1 / 2}-\sigma_{w}^{2}\left(\lambda_{i}^{\left(k^{i}\right)}\right)^{-1}\right)^{+},
$$

where $\mathcal{K}_{i}=\left\{k^{i}\right\}$ and $\nu$ is determined by the power constraint (56), given by

$$
\sqrt{\nu}=\sigma_{w} \frac{1-a^{M_{p}}}{1-a}\left(M_{p} \rho_{p}+\sigma_{w}^{2} \sum_{i:\left|\mathcal{K}_{i}\right|=1}\left(\lambda_{i}^{\left(k^{i}\right)}\right)^{-1}\right)^{-1} .
$$

\section{Suboptimal Power Allocation:}

Consider the high SNR case first, i.e., $\rho_{k^{i}} \lambda_{i j}^{\left(k^{i}\right)} \gg \sigma_{w}^{2}$, where $\boldsymbol{\lambda}_{i}^{\left(k^{i}\right)}=\operatorname{diag}\left(\boldsymbol{\Lambda}_{i}^{\left(k^{i}\right)}\right)$. The cost function (26) can be approximated by

$$
\begin{aligned}
& \sum_{i: \mathcal{K}_{i} \mid=1} \operatorname{tr}\left(\frac{a^{2\left(l M+M_{p}-k^{i}\right)} \sigma_{w}^{2} \mathbf{I}_{N_{r}}}{\rho_{k^{i}} \mathbf{I}_{N_{r}}}\right) \\
& \Rightarrow \min _{\boldsymbol{\rho}} \sum_{i: \mid \mathcal{K}^{\prime}{ }^{\prime}=1} \frac{a^{2\left(l M+M_{p}-k^{i}\right)}}{\rho_{k^{i}}} .
\end{aligned}
$$

This can be solved and the solution is given by (29).

In low SNR $\left(\rho_{k^{i}} \lambda_{i j}^{\left(k^{i}\right)} \ll \sigma_{w}^{2}\right)$, the cost function (26) can be written as

$$
\begin{aligned}
& \sum_{i:\left|\mathcal{K}_{i}\right|=1} \operatorname{tr}\left(\frac{a^{2\left(l M+M_{p}-k^{i}\right)} \sigma_{w}^{2} \boldsymbol{\Lambda}_{i}^{\left(k^{i}\right)}}{\rho_{k^{i}} \boldsymbol{\Lambda}_{i}^{\left(k^{i}\right)}+\sigma_{w}^{2} \mathbf{I}_{N_{r}}}\right) \\
= & \sum_{i:\left|\mathcal{K}_{i}\right|=1} a^{2\left(l M+M_{p}-k^{i}\right)} \sigma_{w}^{2} \operatorname{tr}\left(\mathbf{I}_{N_{r}}+\right. \\
& \left.\frac{\boldsymbol{\Lambda}_{i}^{\left(k^{i}\right)}-\sigma_{w}^{2} \mathbf{I}_{N_{r}}}{\rho_{k^{i}} \boldsymbol{\Lambda}_{i}^{\left(k^{i}\right)}+\sigma_{w}^{2} \mathbf{I}_{N_{r}}}-\frac{\rho_{k^{i}} \boldsymbol{\Lambda}_{i}^{\left(k^{i}\right)}}{\rho_{k^{i}} \boldsymbol{\Lambda}_{i}^{\left(k^{i}\right)}+\sigma_{w}^{2} \mathbf{I}_{N_{r}}}\right) \\
\simeq & \sum_{i:\left|\mathcal{K}_{i}\right|=1} a^{2\left(l M+M_{p}-k^{i}\right)} \operatorname{tr}\left(\boldsymbol{\Lambda}_{i}^{\left(k^{i}\right)}-\rho_{k^{i}} \boldsymbol{\Lambda}_{i}^{\left(k^{i}\right)}\right), \\
\Rightarrow & \max _{\boldsymbol{\rho}} \sum_{i:\left|\mathcal{K}_{i}\right|=1} \rho_{k^{i}} a^{2\left(l M+M_{p}-k^{i}\right)} \operatorname{tr}\left(\boldsymbol{\Lambda}_{i}^{\left(k^{i}\right)}\right) .
\end{aligned}
$$

This can be solved and the solution is given by (30).

\section{REFERENCES}

[1] S. Noh, M. D. Zoltowski, Y. Sung, and D. J. Love, "Optimal pilot beam pattern design for massive MIMO systems," in Proc. Asilomar Conf. Signal, Syst. Comput., Pacific Grove, CA, USA, Nov. 2013.

[2] T. L. Marzetta, "Noncooperative cellular wireless with unlimited numbers of base station antennas," IEEE Trans. Wireless Commun., vol. 9, no. 11, pp. 3590-3600, Nov. 2010.

[3] F. Rusek, D. Persson, B. K. Lau, E. G. Larsson, O. Edfors, F. Tufvesson, and T. L. Marzetta, "Scaling up MIMO: Opportunities and challenges with very large arrays," IEEE Signal Process. Mag., vol. 30, no. 1, pp. 40-60, Jan. 2013.

[4] C. Shepard, H. Yu, N. Anand, L. E. Li, T. L. Marzetta, R. Yang, and L. Zhong, "Argos: Practical many-antenna base stations," in Proc. MobiCom, Istanbul, Turkey, Aug. 2012.

[5] J. Hoydis, S. t. Brink, and M. Debbah, "Massive MIMO in the UL/DL of cellular networks: How many antennas do we need?," IEEE J. Sel. Areas Commun., vol. 31, no. 2, pp. 160-171, Feb. 2013.

[6] P. H. Kuo, H. T. Kung, and P. A. Ting, "Compressive sensing based channel feedback protocols for spatially-correlated massive antenna arrays," in Proc. IEEE WCNC, Paris, France, Apr. 2012, pp. 492-497.
[7] J. Choi, Z. Chance, D. J. Love, and U. Madhow, "Noncoherent trellis coded quantization: A practical limited feedback technique for massive MIMO systems," IEEE Trans. Commun., vol. 61, no. 12, pp. 5016-5029, Dec. 2013.

[8] J. Choi, D. J. Love, and P. Bidigare, "Downlink training techniques for FDD massive MIMO systems: Open-loop and closed-loop training with memory," IEEE J. Sel. Topics Signal Process. [Online]. Available: http://arxiv.org/abs/1309.7712, to be published

[9] J. Nam, J.-Y. Ahn, A. Adhikary, and G. Caire, "Joint spatial division and multiplexing: Realizing massive MIMO gains with limited channel state information," in Proc. IEEE CISS, Pacific Grove, CA, USA, Nov. 2013, pp. 1-6.

[10] K. E. Baddour and N. C. Beaulieu, "Autoregressive modeling for fading channel simulation," IEEE Trans. Wireless Commun., vol. 4 no. 4, pp. 1650-1662, Jul. 2005.

[11] L. Tong, B. M. Sadler, and M. Dong, "Pilot-assisted wireless transmissions: General model, design criteria, and signal processing," IEEE Signal Process. Mag., vol. 21, no. 6, pp. 12-25, Nov. 2004.

[12] H. Yu, Y. Sung, H. Kim, and Y. H. Lee, "Beam tracking for interference alignment in slowly fading MIMO interference channels: A perturbations approach under a linear framework," IEEE Trans. Signal Process., vol. 60, no. 4, pp. 1910-1926, Apr. 2012.

[13] A. M. Sayeed, "Deconstructing multi antenna fading channels," IEEE Trans. Signal Process, vol. 50, no. 10, pp. 2563-2579, Oct. 2002.

[14] D. Shiu, G. J. Foschini, M. J. Gans, and J. M. Kahn, "Fading correlation and its effect on the capacity of multi element antenna systems," IEEE Trans. Commun., vol. 48, no. 3, pp. 502-513, Mar. 2000.

[15] A. Forenza, D. J. Love, and R. W. Heath Jr, "Simplified spatial correlation models for clustered MIMO channels with different array configurations," IEEE Trans. Veh. Technol., vol. 56, no. 4, pp. 1924-1934, Jul. 2007.

[16] A. Adhikary, J. Nam, J.-Y. Ahn, and G. Caire, "Joint spatial division and multiplexing: The large-scale array regime," IEEE Trans. Inf. Theory, vol. 59, no. 10, pp. 6441-6463, Oct. 2013.

[17] M. Dong, L. Tong, and B. M. Sadler, "Optimal insertion of pilot symbols for transmissions over time-varying flat fading channels," IEEE Trans. Signal Process., vol. 52, no. 5, pp. 1403-1418, May 2004.

[18] X. Gao, F. Tufvesson, O. Edfors, and F. Rusek, "Measured propagation characteristics for very-large MIMO at $2.6 \mathrm{GHz}$," in Proc. Asilomar Conf. Signal, Syst. Comput., Pacific Grove, CA, USA, Nov. 2012.

[19] W. C. Jakes, Microwave mobile communication. New York, NY, USA: Wiley, 1974.

[20] S. Stein, "Fading channel issues in system engineering," IEEE J. Sel. Areas Commun., vol. 5, no. 1, pp. 68-89, Feb. 1987.

[21] D. Gerlach and A. Paulraj, "Adaptive transmitting antenna methods for multi path environments," in Proc. IEEE Global Commun. Conf., San Francisco, CA, USA, Nov. 1994, pp. 425-492.

[22] A. Eriksson, P. Stoica, and T. Soderstrom, "On-line subspace algorithms for tracking moving sources," IEEE Trans. Signal Process., vol. 42, no. 9, pp. 2319-2330, Sep. 1994.

[23] L. Tong and S. Perreau, "Multichannel blind identification: from subspace to maximum likelihood methods," Proc. IEEE, vol. 86, no. 10, pp. 1951-1968, Oct. 1998.

[24] S. Noh, Y. Sung, and M. D. Zoltowski, "A new precoder design for blind channel estimation in MIMO-OFDM systems," IEEE Trans. Wireless Commun., accepted for publication.

[25] Y.-C. Liang and F. P. S. Chin, "Downlink channel covariance matrix (DCCM) estimation and its applications in wireless DS-CDMA systems," IEEE J. Sel. Areas Commun., vol. 19, no. 2, pp. 222-232, Feb. 2001.

[26] B. M. Hochwald and T. L. Marzetta, "Adapting a downlink array from uplink measurements," IEEE Trans. Signal Process., vol. 49, no. 3, pp. 642-653, Mar. 2001.

[27] G. C. Raleigh, S. N. Diggavi, V. K. Jones, and A. Paulraj, "A blind adaptive transmit antenna algorithm for wireless communication," in Proc. IEEE Int. Conf. Commun., Seattle, WA, USA, Jun. 1995, pp. 1494-1499.

[28] R. A. Iltis, "Joint estimation of PN code delay and multi path using the extended Kalman filter," IEEE Trans. Commun., vol. 38, no. 10, pp. $1677-1685$, Oct. 1990.

[29] M. K. Tsatsanis, G. B. Giannakis, and G. Zhou, "Estimation and equalization of fading channels with random coefficients," in Proc. IEEE ICASSP, Atlanta, GA, USA, May 1996, pp. 1093-1096.

[30] X. Dai, W. Zhang, J. Xu, J. E. Mitchell, and Y. Yang, "Kalman interpolation filter for channel estimation of LTE downlink in high-mobility environments," EURASIP J. Wireless Commun. Netw., vol. 2012:232, Jul. 2012. 
[31] Z. Liu, A. Hansson, and L. Vandenberghe, "Nuclear norm system identification with missing inputs and outputs," Syst. Control Lett., vol. 62, no. 8, pp. 605-612, Mar. 2013.

[32] Y. Sung, T. E. Sung, B. M. Sadler, and L. Tong, Training for MIMO communications. Space-time wireless systems, 1st ed. Cambridge, U.K.: Cambridge Univ. Press, 2006.

[33] D. J. Love and R. W. Heath Jr, "What is the value of limited feedback for MIMO channels?," IEEE Wireless Commun. Mag., vol. 42, no. 10, pp. 54-59, Oct. 2004.

[34] T. Kailath, A. H. Sayed, and B. Hassibi, Linear estimation. Upper Saddle River, NJ, USA: Prentice-Hall, 2000.

[35] E. Telatar, "Capacity of multi-antenna Gaussian channels," Eur. Trans. Telecommun., vol. 10, no. 6, pp. 585-595, Nov. 1999.

[36] H. Q. Ngo, E. G. Larsson, and T. L. Marzetta, "Energy and spectral efficiency of very large multiuser MIMO systems," IEEE Trans. Commun., vol. 61, no. 4, pp. 1436-1449, Apr. 2013.

[37] H. Yang and T. L. Marzetta, "Performance of conjugate and zero-forcing beamforming in large-scale antenna systems," IEEE J. Sel. Areas Commun., Special Iss. Large-Scale Multiple Antenna Wireless Syst., vol. 31, no. 2, pp. 172-179, Feb. 2013.

[38] B. Hassibi and B. M. Hochwald, "How much training is needed in multiple-antenna wireless links?," IEEE Trans. Inf. Theory, vol. 49, no. 4, pp. 951-963, Apr. 2003.

[39] D. P. Bertsekas, Dynamic programming and Optimal Control, 3rd ed. Belmont, MA, USA: Athena Scientific, 2005

[40] A. Marshall and I. Olkin, Inequalities: Theory of majorization and its applications. New York, NY, USA: Academic, 1979.

[41] T. Cover and J. Thomas, Elements of information theory. New York, NY, USA: Wiley, 1991.

[42] J. H. Kotecha and A. M. Sayeed, "Transmit signal design for optimal estimation of correlated MIMO channels," IEEE Trans. Signal Process., vol. 52, no. 2, pp. 546-557, Feb. 2004.

[43] W. Santipach and M. L. Honig, "Optimization of training and feedback overhead for beamforming over block fading channels," IEEE Trans. Inf. Theory, vol. 56, no. 12, pp. 6103-6115, Dec. 2010.

[44] S. Noh, M. D. Zoltowski, Y. Sung, and D. J. Love, "Training signal design for channel estimation in massive MIMO systems," in Proc. IEEE ICASSP, Florence, Italy, May 2014.

[45] S. Noh, M. D. Zoltowski, and D. J. Love, "Training sequence design for feedback assisted hybrid beamforming in massive MIMO systems," in preparation.

[46] U. Grenander and G. Szegö, Toeplitz forms and their applications. Berkeley, CA, USA: Univ. of California Press, 1958.

[47] Y. Sung, H. V. Poor, and H. Yu, "How much information can one get from a wireless ad hoc sensor network over a correlated random field," IEEE Trans. Inf. Theory, vol. 55, no. 6, pp. 2827-2847, Jun. 2009.

[48] P. J. Brockwell and R. A. Davis, Time series: Theory and methods, 2nd ed. New York, NY, USA: Springer, 1991.

[49] G. Matz, "On non-WSSUS wireless fading channels," IEEE Trans. Wireless Commun., vol. 4, no. 5, pp. 2465-2478, Sep. 2005.

[50] A. Ispas, M. Dörpinghaus, G. Ascheid, and T. Zemen, "Characterization of non-stationary channels using mismatched wiener filtering," IEEE Trans. Signal Process., vol. 61, no. 2, pp. 274-288, Jan. 2013.

[51] K. Fan, "On a theorem of weyl concerning the eigenvalues of linear transformations II," in Proc. Nat. Acad. Sci., Jan. 1950, vol. 36, pp. $31-35$.

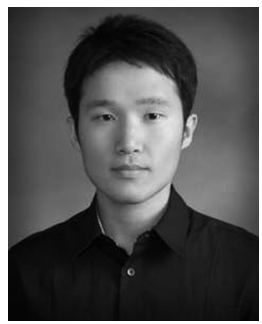

Song Noh (S'08) received the B.S. degree from Soongsil University, Seoul, Korea, in 2008 and the M.S. degree from Korea Advanced Institute of Science and Technology, Daejeon, Korea, in 2010, both in electrical engineering. He is currently working toward the Ph.D. degree in electrical and computer engineering at Purdue University, West Lafayette, IN. His research interests are in the design and analysis of large scale antenna systems and adaptive signal processing for wireless communications.



Michael D. Zoltowski joined the faculty of Purdue University in Fall 1986 where he was named a University Faculty Scholar in 2001. He currently an Endowed Chaired position as the "Thomas J. and Wendy Engibous Professor of Electrical and Computer Engineering," In this capacity he has won several teaching awards, most recently the 2012 Engineering Distance Education Award. He received the 2002 Technical Achievement Award from the IEEE Signal Processing Society (SPS). He served as a 2003 Distinguished Lecturer for the IEEE SPS. $\mathrm{He}$ is a Fellow of IEEE. Dr. Zoltowski is a co-recipient of the IEEE Communications Society 2001 Leonard G. Abraham Prize Paper Award, the IEEE SPS 1991 Paper Award, "The 1998 Fred Ellersick MILCOM Award for Best Paper," and a Best Paper Award at the 2000 IEEE International Symposium on Spread Spectrum Techniques and Applications.

From 1998 to 2001, Dr. Zoltowski served as an elected Member-at-Large of the Board of Governors and Secretary of the IEEE Signal Processing Society (SPS.) From 2003-2005, he served on the Awards Board of the IEEE SPS. and also served as the Area Editor in charge of Feature Articles for the IEEE Signal Processing Magazine. He has served on various SPS Technical Committees. From 2005-2006, he served as Chair of the Technical Committee on Sensor and Multichannel (SAM) Processing. He has served as an Associate Editor for both the IEEE TRANSACTIONS ON SignAL PROCESSING and the IEEE COMMUNICATIONS LETTERS. He was Technical Chair for the 2006 IEEE Sensor Array and Multichannel Workshop. He served as Vice-President for Awards and Membership for the IEEE SPS for 2008-2010. His research interests in lie in all areas of MIMO wireless communications, MIMO radar, and GPS.



Youngchul Sung (S'92-M'93-SM'09) received the B.S. and M.S. degrees from Seoul National University, Seoul, Korea, in electronics engineering in 1993 and 1995, respectively. After working at LG Electronics, Ltd., Seoul, Korea, from 1995 to 2000, he joined the Ph.D. program and received the $\mathrm{Ph} . \mathrm{D}$. degree in electrical and computer engineering from Cornell University, Ithaca, NY, USA, in 2005. From 2005 until 2007, he was a Senior Engineer in the Corporate R\&D Center of Qualcomm, Inc., San Diego, CA, USA, and participated in design of WCDMA base station modem. Since 2007, he has been on the faculty of the Department of Electrical Engineering in KAIST, Daejeon, Korea. His research interests include signal processing for communications, statistical signal processing, asymptotic statistics, and information geometry.

Dr. Sung is currently a member of Signal and Information Processing Theory and Methods (SIPTM) Technical Committee of Asia-Pacific Signal and Information Processing Association (APSIPA), Vice-Chair of IEEE ComSoc Asia-Pacific Board ISC, and was an Associate Editor of the IEEE SIGNAL PROCESSING LETTERS from 2012 to 2014.



David J. Love (S'98-M'05-SM'09) received the B.S. (with highest honors), M.S.E., and Ph.D. degrees in electrical engineering from the University of Texas at Austin in 2000, 2002, and 2004, respectively. During the summers of 2000 and 2002, he was with Texas Instruments, Dallas, TX. Since August 2004, he has been with the School of Electrical and Computer Engineering, Purdue University, West Lafayette, IN, where he is now a Professor and recognized as a University Faculty Scholar.

He has served as an Associate Editor for both the IEEE TRANSACTIONS ON COMMUNICATIONS and the IEEE TRANSACTIONS ON SigNAL PROCESSING, and he has also served as a guest editor for special issues of the IEEE JOURNAL ON SELECTED AREAS IN COMMUNICATIONS and the EURASIP Journal on Wireless Communications and Networking. His research interests are in the design and analysis of communication systems and MIMO array processing. Along with co-authors, he was awarded the 2009 IEEE TRANSACTIONS ON VEHICULAR TECHNOLOGY Jack Neubauer Memorial Award for the best systems paper published in the IEEE TRANSACTIONS ON VEHICULAR TECHNOLOGY in that year and the 2013 IEEE Global Communications Conference (Globecom) Signal Processing for Communications Symposium best paper award. 